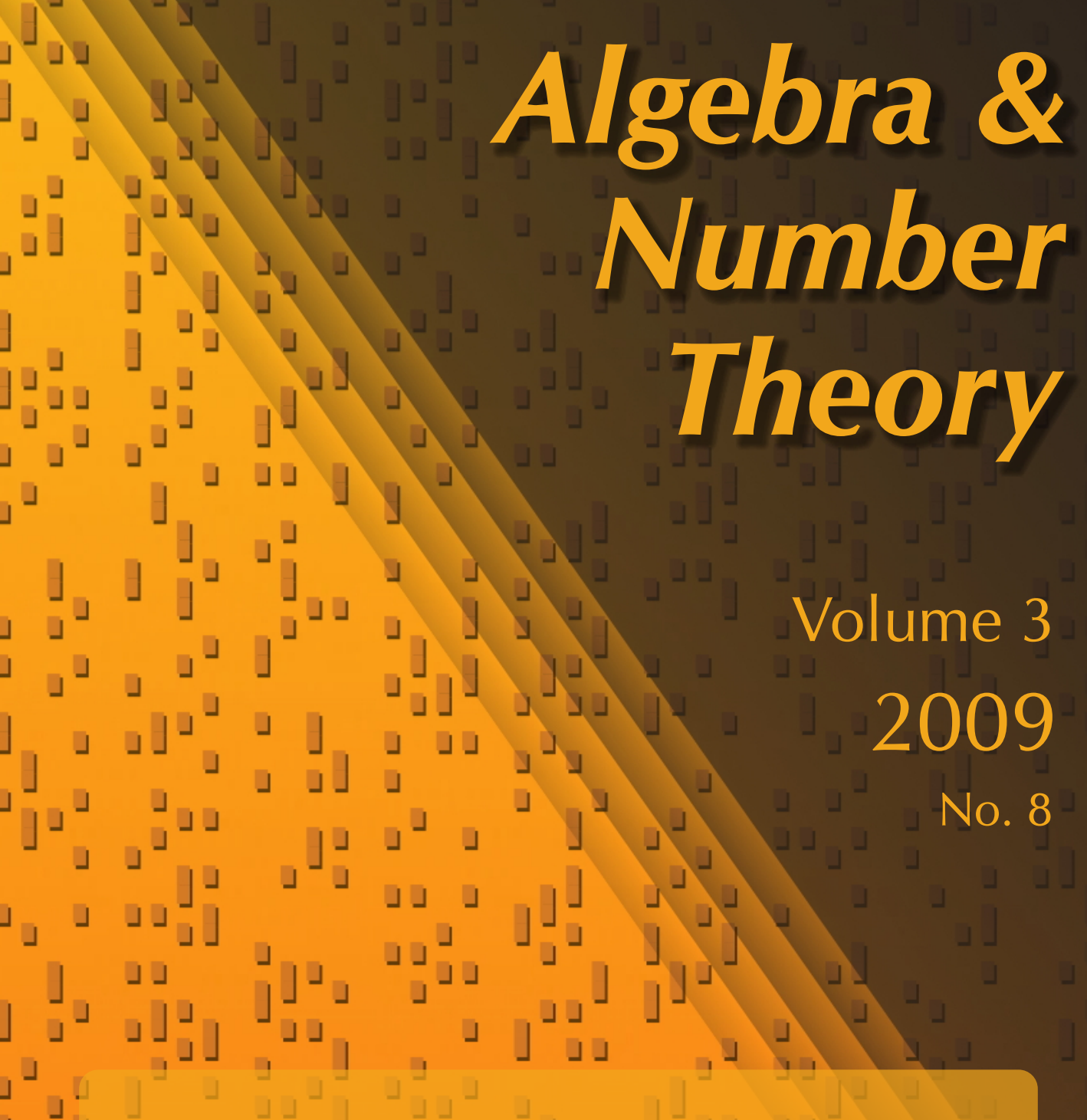

\title{
Centers of graded fusion categories
}

\section{Shlomo Gelaki, Deepak Naidu and Dmitri Nikshych}

」

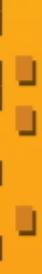




\title{
Centers of graded fusion categories
}

\author{
Shlomo Gelaki, Deepak Naidu and Dmitri Nikshych
}

Let $\mathscr{C}$ be a fusion category faithfully graded by a finite group $G$ and let $\mathscr{D}$ be the trivial component of this grading. The center $\mathscr{L}(\mathscr{C})$ of $\mathscr{C}$ is shown to be canonically equivalent to a $G$-equivariantization of the relative center $\mathscr{L}_{\mathscr{D}}(\mathscr{C})$. We use this result to obtain a criterion for $\mathscr{C}$ to be group-theoretical and apply it to Tambara-Yamagami fusion categories. We also find several new series of modular categories by analyzing the centers of Tambara-Yamagami categories. Finally, we prove a general result about the existence of zeroes in $S$-matrices of weakly integral modular categories.

1. Introduction 959

2. Preliminaries 961

3. The center of a graded fusion category 968

4. The centers of Tambara-Yamagami categories 974

5. Examples of modular categories arising from quadratic forms 980

6. Appendix: Zeroes in $S$-matrices 986

Acknowledgments $\quad 988$

References $\quad 988$

\section{Introduction}

Throughout this paper we work over an algebraically closed field $k$ of characteristic 0 . All categories considered in this paper are finite, abelian, semisimple, and $k$ linear. We freely use the language and basic theory of fusion categories, module categories over them, braided categories, and Frobenius-Perron dimensions [Bakalov and Kirillov 2001; Ostrik 2003; Etingof et al. 2005].

Let $G$ be a finite group. A fusion category $\mathscr{C}$ is $G$-graded if there is a decomposition

$$
\mathscr{b}=\bigoplus_{g \in G} \mathscr{C}_{g}
$$

of $\mathscr{C}$ into a direct sum of full abelian subcategories such that the tensor product of $\mathscr{C}$ maps $\mathscr{b}_{g} \times \mathscr{C}_{h}$ to $\mathscr{C}_{g h}$, for all $g, h \in G$. A $G$-extension of a fusion category $\mathscr{D}$ is a $G$-graded fusion category $\mathscr{C}$ whose trivial component $\mathscr{C}_{e}$, where $e$ is the identity of $G$, is equivalent to $\mathscr{D}$.

MSC2000: primary 16W30; secondary 18D10.

Keywords: fusion categories, braided categories, graded tensor categories. 
Gradings and extensions play an important role in the study and classification of fusion categories. For example, nilpotent fusion categories (that is, those categories that can be obtained from the trivial category by a sequence of group extensions) were studied in [Gelaki and Nikshych 2008]. It was proved in [Etingof et al. 2005] that every fusion category of prime power dimension is nilpotent. Grouptheoretical properties of such categories were studied in [Drinfeld et al. 2007]. Recently, fusion categories of dimension $p^{n} q^{m}$, where $p, q$ are primes, were shown to be Morita equivalent to nilpotent categories [Etingof et al. 2009].

The main goal of this paper is to describe the center $\mathscr{L}(\mathscr{C})$ of a $G$-graded fusion category $\mathscr{C}$ in terms of its trivial component $\mathscr{D}$ (Theorem 3.5) and apply this description to the study of structural properties of $\mathscr{C}$ and the construction of new examples of modular categories.

The organization of the paper is as follows. In Section 2 we recall some basic notions, results, and examples of fusion categories, notably the notions of the relative center of a bimodule category [Majid 1991], group action on a fusion category and crossed product [Tambara 2001], equivariantization and de-equivariantization theory [Arkhipov and Gaitsgory 2003; Bruguières 2000; Gaitsgory 2005; Kirillov 2002; Müger 2000; Drinfeld et al. 2009], and braided $G$-crossed fusion categories [Turaev 2000; 2008].

In Section 3 we study the center $\mathscr{E}(\mathscr{C})$ of a $G$-graded fusion category $\mathscr{C}$. We show that if $\mathscr{D}$ is the trivial component of $\mathscr{C}$, then the relative center $\mathscr{L}_{\mathscr{D}}(\mathscr{C})$ has a canonical structure of a braided $G$-crossed category and there is an equivalence of braided fusion categories $\mathscr{L}_{\mathscr{D}}(\mathscr{C})^{G} \cong \mathscr{L}(\mathscr{C})$ (Theorem 3.5). Thus, the structure of $\mathscr{L}(\mathscr{C})$ can be understood in terms of a smaller and more transparent category $\mathscr{L}_{\mathscr{D}}(\mathscr{C})$. In particular, there is a canonical braided action (studied in detail in [Etingof et al. 2009]) of $G$ on $\mathscr{L}(\mathscr{D})$. In Corollary 3.10 we use this action to prove that $\mathscr{C}$ is grouptheoretical if and only if $\mathscr{L}(\mathscr{D})$ contains a $G$-stable Lagrangian subcategory. As an illustration, we describe the center of a crossed product fusion category $\mathscr{C}=\mathscr{D} \rtimes G$.

We apply the results from Section 4 to the study of Tambara-Yamagami categories [Tambara and Yamagami 1998]. We obtain a convenient description of the centers of such categories as equivariantizations and compute their modular data, that is, $S$ - and $T$-matrices. This computation was previously done in [Izumi 2001] using different techniques. We establish a criterion for a Tambara-Yamagami category to be group-theoretical (Theorem 4.6). We also extend the construction of non-group-theoretical semisimple Hopf algebras from Tambara-Yamagami categories given in [Nikshych 2008].

In Section 5 we construct a series of new modular categories as factors of the centers of Tambara-Yamagami categories. One associates a pair of such categories $\mathscr{E}(q, \pm)$ with any nondegenerate quadratic form $q$ on an abelian group $A$ of odd order. The categories $\mathscr{E}(q, \pm)$ have dimension $4|A|$. They are group-theoretical if 
and only if $A$ contains a Lagrangian subgroup with respect to $q$. We compute the $S$ - and $T$-matrices of $\mathscr{E}(q, \pm)$ and write down several small examples explicitly.

Section 6 is independent from the rest of the paper and contains a general result about existence of zeroes in $S$-matrices of weakly integral modular categories (Theorem 6.1). This is a categorical analogue of a classical result of Burnside in character theory.

\section{Preliminaries}

2A. Dual fusion categories and Morita equivalence. Let $\mathscr{C}$ be a fusion category and let $\mathcal{M}$ be an indecomposable right $\mathscr{C}$-module category $\mathcal{M}$. The category $\mathscr{C}_{\mathcal{M}}^{*}$ of $\mathscr{b}$-module endofunctors of $\mathcal{M}$ is a fusion category, called the dual of $\mathscr{C}$ with respect to $M$ [Etingof et al. 2005; Ostrik 2003].

Following [Müger 2003a], we say that two fusion categories $\mathscr{C}$ and $\mathscr{D}$ are Morita equivalent if $\mathscr{D}$ is equivalent to $\mathscr{C}_{\mathcal{M}}^{*}$, for some indecomposable right $\mathscr{C}$-module category $M$. A fusion category is said to be pointed if all its simple objects are invertible (any such category is equivalent to the category $\operatorname{Vec}_{G}^{\omega}$ of vector spaces graded by a finite group $G$ with the associativity constraint given by a 3-cocycle $\omega \in$ $\left.Z^{3}\left(G, k^{\times}\right)\right)$. A fusion category is called group-theoretical if it is Morita equivalent to a pointed fusion category. See [Ostrik 2003; Etingof et al. 2005; Nikshych 2008] for details of the theory of group-theoretical categories.

\section{B. The center of a bimodule category and the relative center of a fusion cat-} egory. Let $\mathscr{C}$ be a fusion category with unit object $\mathbf{1}$ and associativity constraint $\alpha_{X, Y, Z}:(X \otimes Y) \otimes Z \stackrel{\sim}{\rightarrow} X \otimes(Y \otimes Z)$ and let $M$ be a $\mathscr{C}$-bimodule category.

Definition 2.1. The center of $\mathcal{M}$ is the category $\mathscr{L}_{\mathscr{C}}(\mathcal{M})$ of $\mathscr{C}$-bimodule functors from $\mathscr{b}$ to $M$.

Explicitly, the objects of $\mathscr{L}_{\mathscr{C}}(M)$ are pairs $(M, \gamma)$, where $M$ is an object of $M$ and

$$
\gamma=\left\{\gamma_{X}: X \otimes M \stackrel{\sim}{\rightarrow} M \otimes X\right\}_{X \in \mathscr{C}}
$$

is a natural family of isomorphisms making the diagram

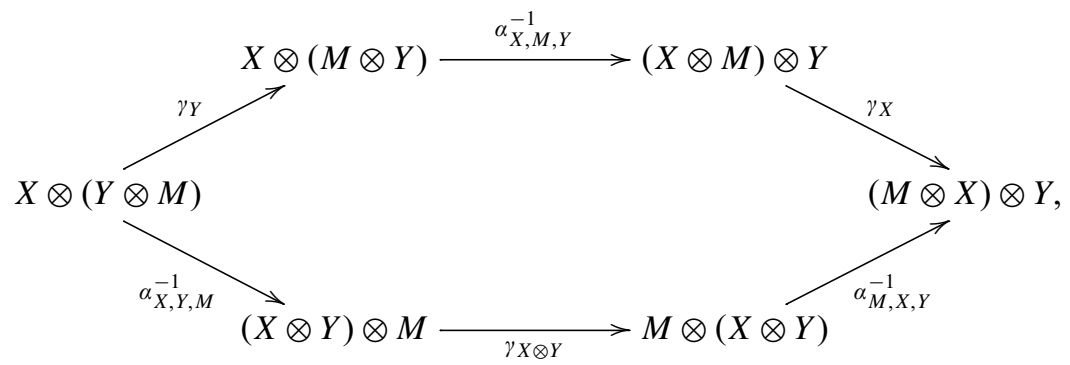

commutative, where the $\alpha$ 's denote the associativity constraints in $\mathcal{M}$. 
Indeed, a $\mathscr{b}$-bimodule functor $F: \mathscr{C} \rightarrow \mathcal{M}$ is completely determined by the pair $\left(F(\mathbf{1}),\left\{\gamma_{X}\right\}_{X \in \mathscr{C}}\right)$, where $\gamma=\left\{\gamma_{X}\right\}_{X \in \mathscr{C}}$ is the collection of isomorphisms

$$
\gamma_{X}: X \otimes F(\mathbf{1}) \stackrel{\sim}{\rightarrow} F(X) \stackrel{\sim}{\rightarrow} F(\mathbf{1}) \otimes X,
$$

coming from the $\mathscr{C}$-bimodule structure on $F$.

We will call the natural family of isomorphisms (1) the central structure of an object $X \in \mathscr{L}_{\mathscr{C}}(M)$.

Remark 2.2. (i) The definition of the center of a bimodule category is parallel to that of the center of a bimodule over a ring.

(ii) We will often suppress the central structure while working with objects of $\mathscr{L}_{\mathscr{C}}(M)$ and refer to $(M, \gamma)$ simply as $M$.

(iii) $\mathscr{L}_{\mathscr{C}}(\mathcal{M})$ is a semisimple abelian category. It has the obvious canonical structure of a $\mathscr{L}(\mathscr{C})$-module category, where $\mathscr{L}(\mathscr{C})$ is the center of $\mathscr{C}$ (see, for example, [Kassel 1995, Section XIII.4] for the definition of $\mathscr{L}(\mathscr{C}))$.

Here is an important special case of this construction. Let $\mathscr{C}$ be a fusion category and let $\mathscr{D} \subset \mathscr{C}$ be a fusion subcategory. Then $\mathscr{C}$ is a $\mathscr{D}$-bimodule category. We will call $\mathscr{L}_{\mathscr{D}}(\mathscr{C})$ the relative center of $\mathscr{C}$.

Remark 2.3. The aforementioned construction of the relative center is a special case of a more general construction considered in [Majid 1991, Definition 3.2 and Theorem 3.3].

It is easy to see that $\mathscr{E}_{\mathscr{D}}(\mathscr{C})$ is a tensor category with tensor product defined as follows. If $(X, \gamma)$ and $\left(X^{\prime}, \gamma^{\prime}\right)$ are objects in $\mathscr{E}_{\mathscr{D}}(\mathscr{C})$ then

$$
(X, \gamma) \otimes\left(X^{\prime}, \gamma^{\prime}\right):=\left(X \otimes X^{\prime}, \tilde{\gamma}\right),
$$

where $\tilde{\gamma}_{V}: V \otimes\left(X \otimes X^{\prime}\right) \stackrel{\sim}{\rightarrow}\left(X \otimes X^{\prime}\right) \otimes V, V \in \mathscr{D}$, is defined by the diagram

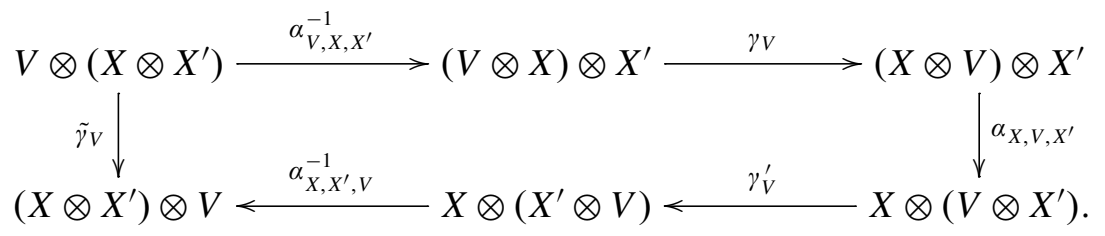

The unit object of $\mathscr{L}_{\mathscr{D}}(\mathscr{C})$ is $(\mathbf{1}$, id $)$. The dual of $(X, \gamma)$ is $\left(X^{*}, \bar{\gamma}\right)$, where $\bar{\gamma}_{V}:=$ $\left(\gamma_{*}\right)^{*}$.

Remark 2.4. Let $\mathscr{C}$ and $\mathscr{D}$ be as above.

(i) $\mathscr{L}_{\mathscr{D}}(\mathscr{C})$ is dual to the fusion category $\mathscr{D} \nabla \mathscr{C}^{\text {rev }}$ (where $\mathscr{C}^{\text {rev }}$ is the fusion category obtained from $\mathscr{C}$ by reversing the tensor product and $\nabla$ is Deligne's tensor product of fusion categories) with respect to its module category $\mathscr{C}$, 
where $\mathscr{D}$ and $\mathscr{C}^{\text {rev }}$ act on $\mathscr{C}$ via the right and left multiplication respectively. In particular, $\mathscr{L}_{\mathscr{D}}(\mathscr{C})$ is a fusion category.

(ii) $\operatorname{FPdim}\left(\mathscr{E}_{\mathscr{D}}(\mathscr{C})\right)=$ FPdim( $(\mathscr{C})$ FPdim(D) , where FPdim denotes the FrobeniusPerron dimension of a category.

(iii) $\mathscr{L}_{\mathscr{C}}(\mathscr{C})$ coincides with the center $\mathscr{L}(\mathscr{C})$ of $\mathscr{C}$. This category has a canonical braiding given by

$$
c_{(X, \gamma),\left(X^{\prime}, \gamma^{\prime}\right)}=\gamma_{X^{\prime}}:(X, \gamma) \otimes\left(X^{\prime}, \gamma^{\prime}\right) \stackrel{\sim}{\rightarrow}\left(X^{\prime}, \gamma^{\prime}\right) \otimes(X, \gamma) .
$$

(iv) There is an obvious forgetful tensor functor:

$$
\mathscr{L}(\mathscr{C}) \mapsto \mathscr{L}_{\mathscr{D}}(\mathscr{C}):(X, \gamma) \mapsto\left(X,\left.\gamma\right|_{\mathscr{D}}\right) .
$$

2C. Centralizers in braided fusion categories. Let $\mathscr{C}$ be a braided fusion category with braiding $c$. Two objects $X$ and $Y$ of $\mathscr{C}$ are said to centralize each other [Müger 2003b] if $c_{Y, X} c_{X, Y}=\mathrm{id}_{X \otimes Y}$.

For any fusion subcategory $\mathscr{D} \subseteq \mathscr{C}$ its centralizer $\mathscr{D}^{\prime}$ is the full fusion subcategory of $\mathscr{C}$ consisting of all objects $X \in \mathscr{C}$ centralizing every object in $\mathscr{D}$. The category $\mathscr{C}$ is said to be nondegenerate if $\mathscr{C}^{\prime}=\mathrm{Vec}$. In this case one has $\mathscr{D}^{\prime \prime}=\mathscr{D}$ [Müger $2003 \mathrm{~b}$ ]. If $\mathscr{C}$ is a premodular category, that is, has a spherical structure, then it is nondegenerate if and only if it is modular.

A braided fusion category $\mathscr{E}$ is called Tannakian if it is equivalent to the representation category $\operatorname{Rep}(G)$ of a finite group $G$ as a braided fusion category. Here $\operatorname{Rep}(G)$ is considered with its standard symmetric braiding. The group $G$ is defined by $\mathscr{E}$ up to an isomorphism [Deligne 1990].

A fusion subcategory $\mathscr{L}$ of a braided fusion category is called Lagrangian if it is Tannakian and $\mathscr{L}=\mathscr{L}^{\prime}$.

Theorem 2.5 [Drinfeld et al. 2007]. A fusion category $\mathscr{C}$ is group-theoretical if and only if $\mathscr{L}(\mathfrak{b})$ contains a Lagrangian subcategory.

2D. Group actions on fusion categories and equivariantization. Let $G$ be a finite group, and let $\underline{G}$ denote the monoidal category whose objects are elements of $G$, whose morphisms are identities, and whose tensor product is given by multiplication in $G$. Recall that an action of $G$ on a fusion category $\mathscr{C}$ is a monoidal functor $\underline{G} \rightarrow \operatorname{Aut}_{\otimes}(\mathscr{C}): g \mapsto T_{g}$. For any $g, h \in G$, let

$$
\gamma_{g, h}=T_{g} \circ T_{h} \simeq T_{g h}
$$

be the isomorphism defining the monoidal structure on the functor $\underline{G} \rightarrow \operatorname{Aut}_{\otimes}(\mathscr{C})$. Definition 2.6. A $G$-equivariant object in $\mathscr{C}$ is a pair $\left(X,\left\{u_{g}\right\}_{g \in G}\right)$ consisting of an object $X$ of $\mathscr{C}$ together with a collection of isomorphisms $u_{g}: T_{g}(X) \simeq X, g \in G$, 
such that the diagram

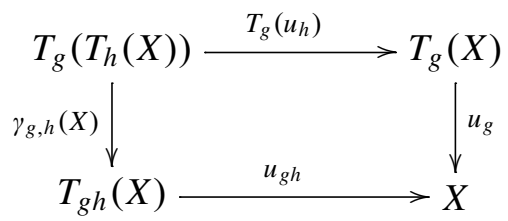

commutes for all $g, h \in G$. One defines morphisms of equivariant objects to be morphisms in $\mathscr{C}$ commuting with $u_{g}, g \in G$.

Equivariant objects in $\mathscr{C}$ form a fusion category, called the equivariantization of $\mathscr{C}$ and denoted by $\mathscr{C}^{G}$ [Tambara 2001; Arkhipov and Gaitsgory 2003; Gaitsgory 2005]. One has FPdim $\left(\mathscr{C}^{G}\right)=|G| \operatorname{FPdim}(\mathscr{C})$.

There is another fusion category that comes from an action of $G$ on $\mathscr{C}$. It is the crossed product category $\mathscr{b} \rtimes G$ defined as follows [Tambara 2001; Nikshych 2008]. As an abelian category, $\mathscr{C} \rtimes G:=\mathscr{C} \otimes \operatorname{Vec}_{G}$, where $\operatorname{Vec}_{G}$ denotes the fusion category of $G$-graded vector spaces. The tensor product in $\mathscr{b} \rtimes G$ is given by

$$
(X \otimes g) \otimes(Y \otimes h):=\left(X \otimes T_{g}(Y)\right) \otimes g h, \quad X, Y \in \mathscr{C}, \quad g, h \in G .
$$

The unit object is $\mathbf{1} \otimes e$ and the associativity and unit constraints come from those of $\mathscr{b}$. Clearly, $\mathscr{b} \rtimes G$ is faithfully $G$-graded with the trivial component $\mathscr{b}$.

As explained in [Nikshych 2008], $\mathscr{C}$ is a right $\mathscr{b} \rtimes G$-module category via

$$
Y \otimes(X \otimes g):=T_{g^{-1}}(Y \otimes X),
$$

and the corresponding dual category $(\mathscr{C} \rtimes G)_{\mathscr{C}}^{*}$ is equivalent to $\mathscr{C}^{G}$. It follows from [Müger 2003a] that there is an equivalence of braided fusion categories

$$
\mathscr{L}(\mathscr{C} \rtimes G) \cong \mathscr{L}\left(\mathscr{C}^{G}\right) .
$$

Let $G$ be a finite group. For any conjugacy class $K$ of $G$ fix a representative $a_{K} \in K$. Let $G_{K}$ denote the centralizer of $a_{K}$ in $G$.

Proposition 2.7. Let $\mathscr{C}=\bigoplus_{g \in G} \mathscr{C}_{g}$ be a $G$-graded fusion category with an action $g \mapsto T_{g}$ of $G$ on $\mathscr{C}_{\text {such }}$ that $T_{g}$ carries $\mathscr{C}_{h}$ to $\mathscr{C}_{g h g^{-1}}$. Let $H:=\left\{g \in G \mid \mathscr{C}_{g} \neq 0\right\}$. There is a bijection between the set of isomorphism classes of simple objects of $\mathscr{C}^{G}$ and pairs $(K, X)$, where $K \subset H$ is a conjugacy class of $G$ and $X$ is a simple $G_{K}$-equivariant object of $\mathscr{C}_{a_{K}}$.

Proof. A simple $G$-equivariant object of $\mathscr{C}$ must be supported on a single conjugacy class $K$. Let $Y=\oplus_{g \in K} Y_{g}$ be such an object. Then $Y_{a_{K}}$ is a simple $G_{K}$-equivariant object.

Conversely, given a $G_{K}$-equivariant object $X$ in $\mathscr{C}_{a_{K}}$ let

$$
Y=\bigoplus_{h} T_{h}(X)
$$


where the summation is taken over the set of representatives of cosets of $G_{K}$ in $G$. It is easy to see that $Y$ acquires the structure of a simple $G$-equivariant object.

Clearly, the two constructions are inverses of each other.

Remark 2.8. The Frobenius-Perron dimension of the simple object corresponding to a pair $(K, X)$ in Proposition 2.7 is $|K| \operatorname{FPdim}(X)$.

2E. De-equivariantization of fusion categories. Let $\mathscr{C}$ be a fusion category. Let $\mathscr{E}=\operatorname{Rep}(G)$ be a Tannakian category along with a braided tensor functor $\mathscr{E} \rightarrow \mathscr{L}(\mathscr{C})$ such that the composition $\mathscr{E} \rightarrow \mathscr{L}(\mathscr{C}) \rightarrow \mathscr{C}$ (where the second arrow is the forgetful functor) is fully faithful. The following construction was introduced in [Bruguières 2000] and [Müger 2000]. Let $A:=\operatorname{Fun}(G)$ be the algebra of functions on $G$. It is a commutative algebra in $\mathscr{E}$ and thus its image is a commutative algebra in $\mathscr{E}(\mathscr{C})$. This fact allows us to view the category $\mathscr{C}_{G}$ of $A$-modules in $\mathscr{C}$ as a fusion category, called de-equivariantization of $\mathscr{C}$. There is a canonical surjective tensor functor

$$
F: \mathscr{b} \rightarrow \mathscr{C}_{G}: X \mapsto A \otimes X
$$

It was explained in [Müger 2000; Drinfeld et al. 2009] that the group $G$ acts on $\mathscr{C}_{G}$ by tensor autoequivalences (this action comes from the action of $G$ on $A$ by right translations). Furthermore, there is a bijection between subcategories of $\mathscr{C}$ containing the image of $\mathscr{E}=\operatorname{Rep}(G)$ and $G$-stable subcategories of $\mathscr{C}_{G}$. This bijection preserves Tannakian subcategories.

The procedures of equivariantization and de-equivariantization are inverses of each other: that is, there are canonical equivalences $\left(\mathscr{C}_{G}\right)^{G} \cong \mathscr{C}$ and $\left(\mathscr{C}^{G}\right)_{G} \cong \mathscr{C}$.

In particular, the construction above applies when $\mathscr{C}$ is a braided fusion category containing a Tannakian subcategory $\mathscr{E}=\operatorname{Rep}(G)$. In this case the braiding of $\mathscr{C}$ gives rise to an additional structure on the de-equivariantization functor (7). Namely, there is natural family of isomorphisms

$$
X \otimes F(Y) \stackrel{\sim}{\rightarrow} F(Y) \otimes X, \quad X \in \mathscr{C}_{G}, Y \in \mathscr{C},
$$

satisfying obvious compatibility conditions. In other words, $F$ can be factored through a braided functor $\mathscr{C} \rightarrow \mathscr{L}\left(\mathscr{C}_{G}\right)$, that is, $F$ is a central functor.

If $\mathscr{E} \subset \mathscr{C}^{\prime}$ then $\mathscr{C}_{G}$ is a braided fusion category with the braiding inherited from that of $\mathscr{C}$. If $\mathscr{E}=\mathscr{C}^{\prime}$, the category $\mathscr{C}_{G}$ is nondegenerate. (In the presence of a spherical structure this category is called the modularization of $\mathscr{C}$ by $\mathscr{E}$ [Bruguières 2000; Müger 2000].)

Remark 2.9. The category $\mathscr{C}_{G}$ is not braided in general. However it does have an additional structure - it is a braided G-crossed fusion category. See next section (2F) for details. 
2F. Braided G-crossed categories. Let $G$ be a finite group. Kirillov [2002] and Müger [2004] found a description of all braided fusion categories $\mathscr{D}$ containing $\operatorname{Rep}(G)$. Namely, they showed that the datum of a braided fusion category $\mathscr{D}$ containing $\operatorname{Rep}(G)$ is equivalent to the datum of a braided $G$-crossed category $\mathscr{C}$; see Theorem 2.12. The notion of a braided $G$-crossed category is due to Turaev [2000; 2008] and is recalled below.

Definition 2.10. A braided $G$-crossed fusion category is a fusion category $\mathscr{C}$ equipped with (i) a (not necessarily faithful) grading $\mathscr{C}=\bigoplus_{g \in G} \mathscr{C}_{g}$, (ii) an action $g \mapsto T_{g}$ of $G$ on $\mathscr{C}$ such that $T_{g}\left(\mathscr{C}_{h}\right) \subset \mathscr{C}_{g h g^{-1}}$, and (iii) a natural collection of isomorphisms

$$
c_{X, Y}: X \otimes Y \simeq T_{g}(Y) \otimes X, \quad X \in \mathscr{C}_{g}, g \in G \text { and } Y \in \mathscr{C},
$$

called the $G$-braiding. These structures are required to satisfy certain compatibility conditions, which we now state. Let $\gamma_{g, h}: T_{g} T_{h} \stackrel{\sim}{\rightarrow} T_{g h}$ denote the tensor structure of the functor $g \mapsto T_{g}$ and $\mu_{g}$ the tensor structure of $T_{g}$.

(a) The diagram

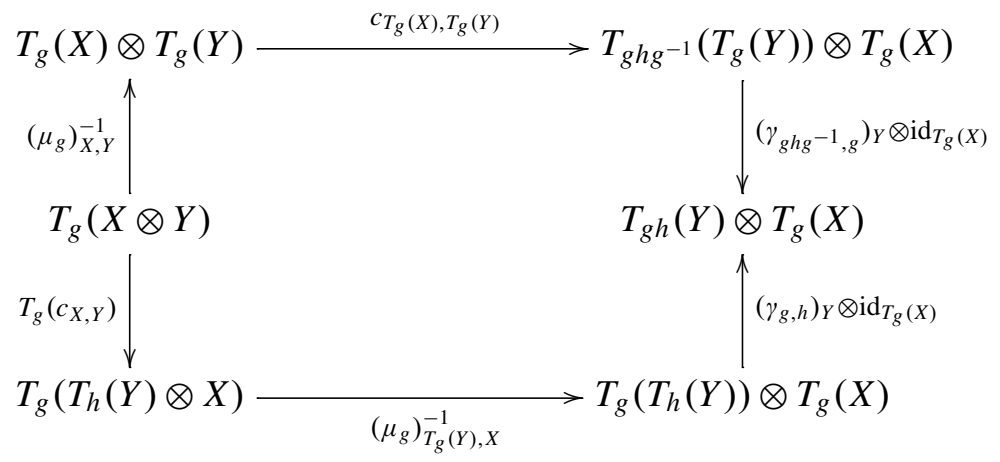

commutes for all $g, h \in G$ and objects $X \in \mathscr{C}_{h}, Y \in \mathscr{C}$.

(b) The diagram

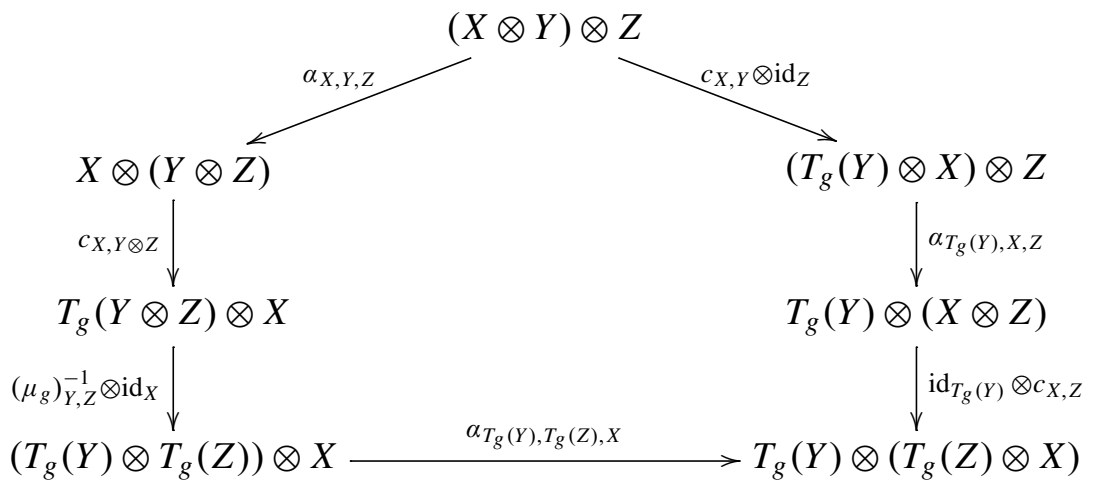

commutes for all $g \in G$ and objects $X \in \mathscr{C}_{g}, Y, Z \in \mathscr{C}$. 
(c) The diagram

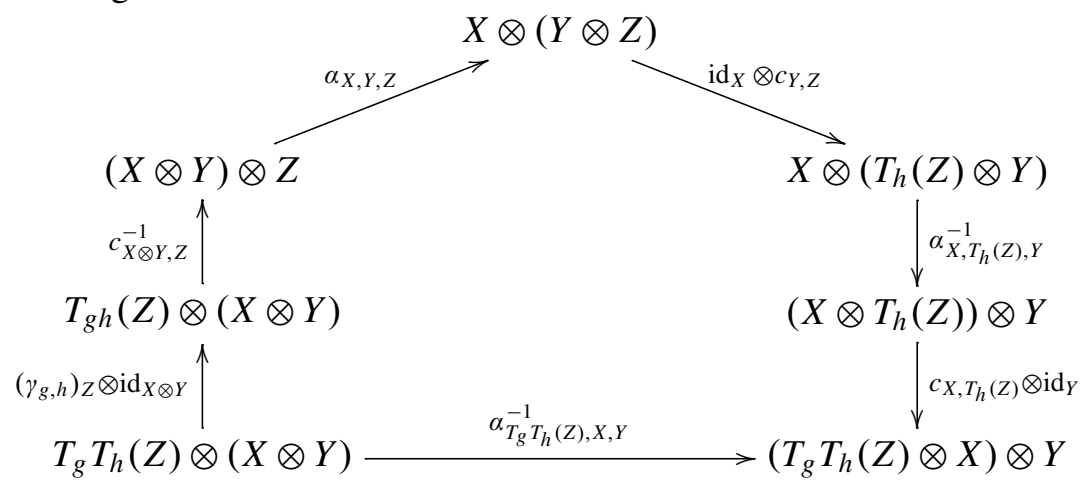

commutes for all $g, h \in G$ and objects $X \in \mathscr{C}_{g}, Y \in \mathscr{C}_{h}, Z \in \mathscr{C}$.

Remark 2.11. The trivial component $\mathscr{C}_{e}$ of a braided $G$-crossed fusion category $\mathscr{C}$ is a braided fusion category with the action of $G$ by braided autoequivalences. This can be seen by taking $X, Y \in \mathscr{C}_{e}$ in diagrams (10)-(12).

Theorem 2.12 ([Kirillov 2002; Müger 2004]). The equivariantization and deequivariantization constructions establish a bijection between the set of equivalence classes of $G$-crossed braided fusion categories and the set of equivalence classes of braided fusion categories containing $\operatorname{Rep}(G)$ as a symmetric fusion subcategory.

We shall now sketch the proof of this theorem. An alternative approach is given in [Drinfeld et al. 2009].

Suppose $\mathscr{C}$ is a braided $G$-crossed fusion category. We define a braiding $\tilde{c}$ on its equivariantization $\mathscr{C}^{G}$ as follows.

Let $\left(X,\left\{u_{g}\right\}_{g \in G}\right)$ and $\left(Y,\left\{v_{g}\right\}_{g \in G}\right)$ be objects in $\mathscr{C}^{G}$. Let $X=\oplus_{g \in G} X_{g}$ be a decomposition of $X$ with respect to the grading of $\mathscr{C}$. Define an isomorphism

$\tilde{c}_{X, Y}: X \otimes Y=\bigoplus_{g \in G} X_{g} \otimes Y \stackrel{\oplus c_{X_{g}, Y}}{\longrightarrow} \bigoplus_{g \in G} T_{g}(Y) \otimes X_{g} \stackrel{\oplus v_{g} \otimes \mathrm{id}_{X_{g}}}{\longrightarrow} \bigoplus_{g \in G} Y \otimes X_{g}=Y \otimes X$.

It follows from condition (a) of Definition 2.10 that $\tilde{c}_{X, Y}$ respects the equivariant structures, that is, it is an isomorphism in $\mathscr{C}^{G}$. Its naturality is clear. The fact that $\tilde{c}$ is a braiding on $\mathscr{C}^{G}$ (that is, the hexagon axioms) follows from the commutativity of diagrams (11) and (12). It is easy to check that $\tilde{c}$ restricts to the standard braiding on $\operatorname{Rep}(G)=\operatorname{Vec}^{G} \subset \mathscr{C}^{G}$. Hence, $\mathscr{C}^{G}$ contains a Tannakian subcategory $\operatorname{Rep}(G)$.

Conversely, let $\mathscr{C}$ be a braided fusion category with braiding $c$ containing a Tannakian subcategory $\operatorname{Rep}(G)$. The restriction of the de-equivariantization functor $F$ from (7) on $\operatorname{Rep}(G)$ is isomorphic to the fiber functor $\operatorname{Rep}(G) \rightarrow$ Vec. Hence for any object $X$ in $\mathscr{C}_{G}$ and any object $V$ in $\operatorname{Rep}(G)$ we have an automorphism of 
$F(V) \otimes X$ defined as the composition

$$
F(V) \otimes X \stackrel{\sim}{\rightarrow} X \otimes F(V) \stackrel{\sim}{\rightarrow} F(V) \otimes X,
$$

where the first isomorphism comes from the fact that $F(V) \in \mathrm{Vec}$ and the second one is (8).

When $X$ is simple we have an isomorphism $\operatorname{Aut}_{\mathscr{C}}(F(V) \otimes X) \cong \operatorname{Aut}_{\mathrm{Vec}}(F(V))$, hence we obtain a tensor automorphism $i_{X}$ of $\left.F\right|_{\operatorname{Rep}(G)}$. Since $\operatorname{Aut}_{\otimes}\left(\left.F\right|_{\operatorname{Rep}(G)}\right) \cong G$ we have an assignment $X \mapsto i_{X} \in G$. The hexagon axiom of braiding implies that this assignment is multiplicative, that is, that $i_{Z}=i_{X} i_{Y}$ for any simple object $Z$ contained in $X \otimes Y$. Thus, it defines a $G$-grading on $\mathscr{C}$ :

$$
\mathscr{b}=\bigoplus_{g \in G} \mathscr{C}_{g}, \quad \text { where } \mathcal{O}\left(\mathscr{C}_{g}\right)=\left\{X \in \mathcal{O}(\mathscr{C}) \mid i_{X}=g\right\} .
$$

It is straightforward to check that $i_{T_{g}(X)}=g h g^{-1}$ whenever $i_{X}=h$.

Finally, to construct a $G$-crossed braiding on $\mathscr{C}$, observe that $\mathscr{C}$ and $\mathscr{C}^{\text {rev }}$ are embedded into the crossed product category $\mathscr{b} \rtimes G=\left(\mathscr{C}^{G}\right)_{\mathscr{C}}^{*}$ as subcategories $\mathscr{C}_{\text {left }}$ and $\mathscr{C}_{\text {right }}$, consisting, respectively, of functors of left and right multiplications by objects of $\mathscr{C}$. Clearly, there is a natural family of isomorphisms

$$
X \otimes Y \stackrel{\sim}{\rightarrow} Y \otimes X, \quad \text { with } X \in \mathscr{C}_{\text {left }} \text { and } Y \in \mathscr{C}_{\text {right }},
$$

satisfying obvious compatibility conditions. Note that $\mathscr{C}_{\text {left }}$ is identified with the diagonal subcategory of $\mathscr{C} \rtimes G$ spanned by objects $X \otimes g, X \in \mathscr{C}_{g}, g \in G$, and $\mathscr{C}_{\text {right }}$ is identified with the trivial component subcategory $\mathscr{C} \otimes e$. Using (6) we conclude that isomorphisms (16) give rise to a $G$-crossed braiding on $\mathscr{b}$.

One can check that the two constructions above (from braided fusion categories containing $\operatorname{Rep}(G)$ to braided $G$-crossed categories and vice versa) are inverses of each other; see [Kirillov 2002; Müger 2004; Drinfeld et al. 2009] for details.

Remark 2.13. Let $\mathscr{C}=\oplus_{g \in G} \mathscr{C}_{g}$ be a braided $G$-crossed fusion category. It was shown in [Drinfeld et al. 2009] that the braided category $\mathscr{C}^{G}$ is nondegenerate if and only if $\mathscr{C}_{e}$ is nondegenerate and the $G$-grading of $\mathscr{C}$ is faithful.

\section{The center of a graded fusion category}

Let $G$ be a finite group and let $\mathscr{D}$ be a fusion category. Throughout this section $\mathscr{C}$ will denote a fusion category with a faithful $G$-grading, whose trivial component is $\mathscr{D}$; that is, $\mathscr{C}$ is a $G$-extension of $\mathscr{D}$ :

$$
\mathscr{b}=\bigoplus_{g \in G} \mathscr{C}_{g}, \quad \mathscr{C}_{e}=\mathscr{D} .
$$


In what follows we consider only faithful gradings: that is, those such that $\mathscr{C}_{g} \neq 0$ for all $g \in G$. An object of $\mathscr{C}$ contained in $\mathscr{C}_{g}$ will be called homogeneous of degree $g$.

Our goal is to describe the center $\mathscr{L}(\mathscr{C})$ as an equivariantization of the relative center $\mathscr{L}_{\mathscr{D}}(\mathscr{C})$ defined in Section $2 \mathrm{~B}$.

3A. The relative center $\mathscr{L}_{\mathscr{D}}(\mathscr{C})$ as a braided $G$-crossed category. Let us define a canonical braided $G$-crossed category structure on $\mathscr{L}_{\mathscr{D}}(\mathscr{C})$.

First of all, there is an obvious faithful $G$-grading on $\mathscr{L}_{\mathscr{D}}(\mathscr{C})$ :

$$
\mathscr{L}_{\mathscr{D}}(\mathscr{C})=\bigoplus_{g \in G} \mathscr{L}_{\mathscr{D}}\left(\mathscr{C}_{g}\right) \text {. }
$$

Indeed, it is clear that for every simple object $X$ of $\mathscr{L}_{\mathscr{D}}(\mathscr{C})$ the forgetful image of $X$ in $\mathscr{C}$ must be homogeneous.

We now define the action of $G$ on $\mathscr{L}_{\mathscr{D}}(\mathscr{C})$. Take $g, h \in G$. Let Fun $\mathscr{D}_{\mathscr{D}}$ rev $\left(\mathscr{C}_{g}, \mathscr{C}_{h}\right)$ denote the category of $\mathscr{D}$-bimodule functors from $\mathscr{C}_{g}$ to $\mathscr{C}_{h}$. Clearly, it is a $\mathscr{L}(\mathscr{D})$ bimodule category.

Proposition 3.1. Let $g, h \in G$. The functors

$$
\begin{aligned}
& L_{g, h}: \mathscr{L}_{\mathscr{D}}\left(\mathscr{C}_{h}\right) \stackrel{\sim}{\rightarrow} \operatorname{Fun}_{\mathscr{D} \otimes \mathscr{D} r e v}\left(\mathscr{C}_{g}, \mathscr{C}_{h g}\right): Z \mapsto Z \otimes ?, \\
& R_{g, h}: \mathscr{L}_{\mathscr{D}}\left(\mathscr{C}_{h}\right) \stackrel{\sim}{\rightarrow} \operatorname{Fun}_{\mathscr{D} \otimes \mathscr{D} \text { rev }}\left(\mathscr{C}_{g}, \mathscr{C}_{g h}\right): Z \mapsto ? \otimes Z .
\end{aligned}
$$

are equivalences of $\mathscr{L}(\mathfrak{D})$-bimodule categories.

Proof. We prove that (19) is an equivalence. Let Fun $\left(\mathscr{C}_{g}, \mathscr{C}_{h g}\right)$ be the category of right $\mathscr{D}$-module functors from $\mathscr{C}_{g}$ to $\mathscr{C}_{h g}$. It suffices to prove that

$$
M_{g, h}: \mathscr{C}_{h} \rightarrow \operatorname{Fun}_{\mathscr{D}}\left(\mathscr{C}_{g}, \mathscr{C}_{h g}\right): X \mapsto X \otimes ?
$$

is an equivalence. Indeed, $\mathscr{D}$-bimodule functor structures on $M_{g, h}(X)$ for $X \in \mathscr{C}_{h}$ are in bijection with central structures on $X$.

For every $g \in G$ choose a simple object $X_{g} \in \mathscr{C}_{g}$. Then $A_{g}:=X_{g} \otimes X_{g}^{*}$ is an algebra in $\mathscr{D}$. It follows from [Ostrik 2003, Theorem 1] that the functor $Y \mapsto Y \otimes X_{g}^{*}$ is a left $\mathscr{b}$-module category equivalence between $\mathscr{C}$ and the category of right $A_{g}$ modules in $\mathscr{C}$. Since $Y \otimes X_{g}^{*}$ belongs to $\mathscr{D}$ if and only if $Y$ is in $\mathscr{C}_{g}$ we see that the functor above restricts to a left $\mathscr{D}$-module category equivalence between $\mathscr{b}_{g}$ and the category of right $A_{g}$-modules in $\mathscr{D}$. There are also similar equivalences of right module categories.

It follows that for all $g, h \in G$ there is an equivalence

$$
Y \mapsto X_{g} \otimes Y \otimes X_{h g}^{*}
$$

between $\mathscr{C}$ and the category of $\left(A_{g}-A_{h g}\right)$-bimodules in $\mathscr{C}$. The right-hand side of (22) belongs to $\mathscr{D}$ if and only if $Y$ is in $\mathscr{C}_{h}$. Hence, (22) restricts to an equivalence 
between $\mathscr{C}_{h}$ and the category of $\left(A_{g}-A_{h g}\right)$-bimodules in $\mathscr{D}$. The latter category is identified with the category of right $\mathscr{D}$-module functors between the categories of right $A_{g}$-modules and $A_{h g}$-modules in $\mathscr{D}$, that is, with Fun $\mathscr{D}_{\mathscr{D}}\left(\mathscr{C}_{g}, \mathscr{C}_{h g}\right)$. It is easy to see that upon this identification the restriction of equivalence (22) to $\mathscr{C}_{h}$ coincides with (21).

The proof of the equivalence (20) is completely similar.

We define tensor functors

$$
T_{g, h}:=L_{g, g h g^{-1}}^{-1} R_{g, h}: \mathscr{L}_{\mathscr{D}}\left(\mathscr{C}_{h}\right) \rightarrow \mathscr{L}_{\mathscr{D}}\left(\mathscr{C}_{g h g^{-1}}\right), \quad g, h \in G,
$$

and set

$$
T_{g}:=\bigoplus_{h \in G} T_{g, h}: \mathscr{L}_{\mathscr{D}}(\mathscr{C}) \rightarrow \mathscr{L}_{\mathscr{D}}(\mathscr{C})
$$

The definition of $T_{g}$ along with Proposition 3.1 give rise to the following natural isomorphism of $\mathscr{D}$-bimodule functors from $\mathscr{C}_{g}$ to $\mathscr{C}$ :

$$
c_{-, Y}: ? \otimes Y \stackrel{\sim}{\rightarrow} T_{g}(Y) \otimes ? .
$$

It translates to a natural family of isomorphisms

$$
c_{X, Y}: X \otimes Y \stackrel{\sim}{\rightarrow} T_{g}(Y) \otimes X, \quad X \in \mathscr{C}_{g}, Y \in \mathscr{L}_{\mathscr{D}}(\mathscr{C}), g \in G,
$$

satisfying natural compatibility conditions corresponding to the $\mathscr{D}$-bimodule structure on (25). Since the grading (18) is faithful, we have $T_{g}\left(\mathscr{L}_{\mathscr{D}}\left(\mathscr{C}_{h}\right)\right) \subset \mathscr{E}_{\mathscr{D}}\left(\mathscr{C}_{g h g^{-1}}\right)$.

Take $X_{1} \in \mathscr{C}_{g_{1}}, X_{2} \in \mathscr{C}_{g_{2}}$ and set $X=X_{1} \otimes X_{2}$ in (26). We obtain a natural isomorphism

$$
T_{g_{1}} T_{g_{2}}(Y) \otimes X_{1} \otimes X_{2} \stackrel{\sim}{\rightarrow} T_{g_{1} g_{2}}(Y) \otimes X_{1} \otimes X_{2}
$$

Since every object $Z \in \mathscr{C}_{g_{1} g_{2}}$ is contained in $X_{1} \otimes X_{2}$ for some $X_{1} \in \mathscr{C}_{g_{1}}, X_{2} \in \mathscr{C}_{g_{2}}$, using naturality of (27) we obtain a natural isomorphism

$$
T_{g_{1}} T_{g_{2}}(Y) \otimes Z \stackrel{\sim}{\rightarrow} T_{g_{1} g_{2}}(Y) \otimes Z, \quad Z \in \mathscr{C}_{g_{1} g_{2}},
$$

of $\mathscr{D}$-bimodule functors $T_{g_{1}} T_{g_{2}}(Y) \otimes$ ? and $T_{g_{1} g_{2}}(Y) \otimes$ ?. By Proposition 3.1 this gives an isomorphism $T_{g_{1}} T_{g_{2}}(Y) \stackrel{\sim}{\rightarrow} T_{g_{1} g_{2}}(Y), Y \in \mathscr{E}_{\mathscr{D}}(\mathscr{C})$, that is, an isomorphism of functors $T_{g_{1}} T_{g_{2}} \stackrel{\sim}{\rightarrow} T_{g_{1} g_{2}}$. Thus, the assignment $g \mapsto T_{g}$ is an action of $G$ on $\mathscr{L}_{\mathscr{D}}(\mathscr{C})$ by tensor autoequivalences.

Suppose that $X$ is an object in $\mathscr{L}\left(\mathscr{C}_{g}\right)$. Then both sides of (26) have structure of objects in $\mathscr{L}_{\mathscr{D}}(\mathscr{C})$ obtained by composing central structures of $X$ and $Y$.

Lemma 3.2. Isomorphisms (26) define a G-braiding on $\mathscr{L}_{\mathscr{D}}(\mathscr{C})$. 
Proof. That isomorphisms (26) are indeed morphisms in $\mathscr{\mathscr { L }} \mathscr{\mathscr { O }}(\mathscr{C})$ follows from commutativity of the diagram

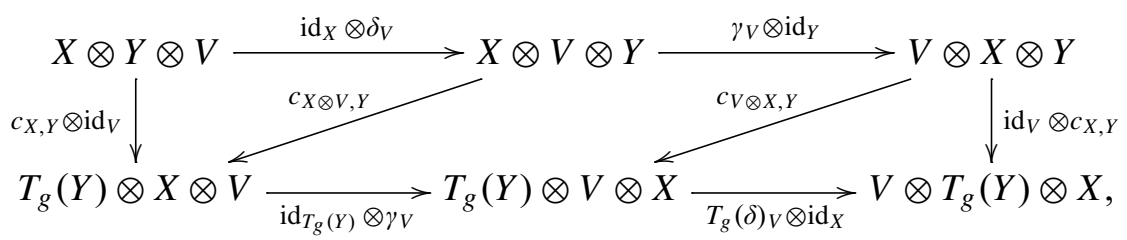

where $(X, \gamma) \in \mathscr{L}_{\mathscr{D}}\left(\mathscr{C}_{g}\right),(Y, \delta) \in \mathscr{L}_{\mathscr{D}}(\mathscr{C})$, and $V \in \mathscr{D}$. Indeed, the parallelogram in the middle commutes by naturality of $c$, and the two triangular faces commute since the natural isomorphism (25) is an isomorphism of D-bimodule functors.

It is straightforward to check that isomorphisms $c_{X, Y}$ satisfy the compatibility conditions of Definition 2.10.

The constructions and arguments above prove the following theorem.

Theorem 3.3. Let $G$ be a finite group and let $\mathscr{C}$ be a fusion category with a faithful $G$-grading whose trivial component is $\mathscr{D}$. The relative center $\mathscr{L}_{\mathscr{D}}(\mathscr{C})$ has a canonical structure of a braided $G$-crossed category.

Remark 3.4. In particular, to every $G$-extension of a fusion category $\mathscr{D}$ we assigned an action of $G$ by braided autoequivalences of $\mathscr{L}(\mathscr{D})$. This assignment is studied in detail in [Etingof et al. 2009].

3B. The center $\mathscr{L}(\mathscr{C})$ as an equivariantization. As before, let $G$ be a finite group and let $\mathscr{C}$ be a fusion category with a faithful $G$-grading (17). Let $\mathscr{L}_{\mathscr{D}}(\mathscr{C})$ be the braided $G$-crossed category constructed in Section 3A.

Theorem 3.5. There is an equivalence of braided fusion categories

$$
\mathscr{L}_{\mathscr{D}}(\mathscr{C})^{G} \stackrel{\mathscr{\sim}}{\rightarrow}(\mathscr{C}) .
$$

Proof. We see from (26) that a $G$-equivariant object in $\mathscr{L}_{\mathscr{D}}(\mathscr{C})$ has a structure of a central object in $\mathscr{C}$ defined as in (13). It follows from definitions that the corresponding tensor functor $\mathscr{L}_{\mathscr{D}}(\mathscr{C})^{G} \rightarrow \mathscr{L}(\mathscr{C})$ is braided.

Conversely, given an object $Y$ in $\mathscr{E}(\mathscr{C})$, consider its forgetful image $\tilde{Y}$ in $\mathscr{E} \mathscr{D}(\mathscr{C})$. Combining the central structure of $Y$ with isomorphism (26) we obtain a family of isomorphisms

$$
\tilde{Y} \otimes X \stackrel{\sim}{\rightarrow} T_{g}(\tilde{Y}) \otimes X, \quad X \in \mathscr{C}_{g}, g \in G,
$$

which gives rise to the isomorphism of $\mathscr{D}$-bimodule functors $\tilde{Y} \otimes ? \stackrel{\sim}{\rightarrow} T_{g}(\tilde{Y}) \otimes$ ? : $\mathscr{C}_{g} \rightarrow \mathscr{C}$. By Proposition 3.1 we obtain a natural isomorphism $\tilde{Y} \stackrel{\sim}{\rightarrow} T_{g}(\tilde{Y})$ and, hence, a $G$-equivariant structure on $\tilde{Y}$. Thus, we have a tensor functor $\mathscr{L}(\mathscr{C}) \rightarrow$ $\mathscr{L}_{\mathscr{D}}(\mathscr{C})^{G}$. It is clear that the two functors are quasiinverses of each other. 
We describe the Tannakian subcategory $\mathscr{E} \cong \operatorname{Rep}(G) \subset \mathscr{L}(\mathscr{C})$ corresponding to equivalence (30). For any representation $\pi: G \rightarrow G L(V)$ of the grading group $G$, consider an object $I_{\pi}$ in $\mathscr{L}(\mathscr{C})$ where $I_{\pi}=V \otimes \mathbf{1}$ as an object of $\mathscr{C}$ with the permutation isomorphism

$$
c_{I_{\pi}, X}:=\pi(g) \otimes \operatorname{id}_{X}: I_{\pi} \otimes X \cong X \otimes I_{\pi}, \quad \text { when } X \in \mathscr{C}_{g} .
$$

Then $\mathscr{E}$ is the subcategory of $\mathscr{L}(\mathscr{C})$ consisting of objects $I_{\pi}$, where $\pi$ runs through all finite-dimensional representations of $G$.

Remark 3.6. Here is another description of the subcategory $\mathscr{E}$ : it consists of all objects in $\mathscr{L}(\mathscr{C})$ sent to Vec by the forgetful functor $\mathscr{L}(\mathscr{C}) \rightarrow \mathscr{L}_{\mathscr{D}}(\mathscr{C})$.

Corollary 3.7. Let $\mathscr{C}$ be a faithfully $G$-graded fusion category with the trivial component $\mathscr{D}$. Let $\mathscr{E}=\operatorname{Rep}(G) \subset \mathscr{L}(\mathscr{C})$ be the Tannakian subcategory constructed above. Then the de-equivariantization category $\left(\mathscr{E}^{\prime}\right)_{G}$ is braided tensor equivalent to $\mathscr{L}(\mathscr{D})$.

Proof. The statement follows from Theorem 3.5 since $\left(\mathscr{C}^{\prime}\right)_{G}$ is the trivial component of the grading of $\mathscr{L}(\mathscr{C})_{G}=\mathscr{L}_{\mathscr{D}}(\mathscr{C})$.

Remark 3.8. The assignment above

$$
\{G \text {-extensions of } \mathscr{D}\} \mapsto\{\text { braided } G \text {-crossed extensions of } \mathscr{L}(\mathscr{D})\}
$$

can be thought of as an analogue of the center construction for $G$-extensions.

Next, we describe simple objects of $\mathscr{E}(\mathscr{C})$. For any conjugacy class $K$ in $G$ fix a representative $a_{K} \in K$. Let $G_{K}$ denote the centralizer of $a_{K}$ in $G$. Note that the action (24) of $G$ on $\mathscr{L}_{\mathscr{D}}(\mathscr{C})$ restricts to the action of $G_{K}$ on $\mathscr{L}_{\mathscr{D}}\left(\mathscr{C}_{a_{K}}\right)$.

Proposition 3.9. There is a bijection between the set of isomorphism classes of simple objects of $\mathscr{L}(\mathscr{C})$ and pairs $(K, X)$, where $K$ is a conjugacy class of $G$ and $X$ is a simple $G_{K}$-equivariant object of $\mathscr{E}_{\mathscr{D}}\left(\mathscr{C}_{a_{K}}\right)$.

Proof. By Theorem 3.5 we have $\mathscr{L}(\mathscr{C}) \simeq \mathscr{L}_{\mathscr{D}}(\mathscr{C})^{G}$, so the stated parameterization is immediate from the description of simple objects of the equivariantization category given in Proposition 2.7.

3C. A criterion for a graded fusion category to be group-theoretical. We have seen in Corollary 3.7 that $\mathscr{L}(\mathscr{C})$ contains a Tannakian subcategory $\mathscr{E}=\operatorname{Rep}(G)$ such that the de-equivariantization $\left(\mathscr{E}^{\prime}\right)_{G}$ is braided equivalent to $\mathscr{L}(\mathscr{D})$, where $\mathscr{D}$ is the trivial component of $\mathscr{C}$. Furthermore, by Remark 2.11, there is a canonical action of $G$ on $\mathscr{L}(\mathscr{D})$, by braided autoequivalences. By [Drinfeld et al. 2009], Tannakian subcategories of $\mathscr{L}(\mathscr{C})$ containing $\mathscr{E}$ bijectively correspond to $G$-stable Tannakian subcategories of $\left(\mathscr{E}^{\prime}\right)_{G} \simeq \mathscr{L}(\mathscr{D})$. Combining this observation with Theorem 2.5(ii) we obtain the following criterion. 
Corollary 3.10. A graded fusion category $\mathscr{C}=\bigoplus_{g \in G} \mathscr{C}_{g}, \mathscr{C}_{e}=\mathscr{D}$, is grouptheoretical if and only if $\mathscr{L}(\mathscr{D})$ contains a $G$-stable Lagrangian subcategory.

Corollary 3.10 will be useful in Section 4D, where we characterize grouptheoretical Tambara-Yamagami categories.

We can specialize Corollary 3.10 to equivariantization categories. Let $G$ be a finite group acting on a fusion category $\mathscr{C}$. The equivariantization $\mathscr{C}^{G}$ is Morita equivalent to the crossed product category $\mathscr{b} \rtimes G$ (see Section 2D). Therefore, $\mathscr{L}\left(\mathscr{C}^{G}\right) \cong \mathscr{L}(\mathscr{C} \rtimes G)$. Clearly, the trivial component of $\mathscr{L}(\mathscr{C} \rtimes G)_{G}$ is $\mathscr{L}(\mathscr{C})$ and the canonical action of $G$ on $\mathscr{E}(\mathscr{C})$ is induced from the action of $G$ on $\mathscr{C}$ in an obvious way.

Corollary 3.11. The equivariantization $\mathscr{C}^{G}$ is group-theoretical if and only if there exists a $G$-stable Lagrangian subcategory of $\mathscr{E}(\mathscr{C})$.

Remark 3.12. Let $G$ act on $\mathscr{C}$ as before. One can check (independently from the results of this section) that the $G$-set of Lagrangian subcategories of $\mathscr{L}(\mathscr{C})$ is isomorphic to the $G$-set consisting of indecomposable $\mathscr{C}$-module categories $M$ such that the dual category $\mathscr{C}_{\mathcal{M}}^{*}$ is pointed. This isomorphism is given by the map constructed in [Naidu and Nikshych 2008, Theorem 4.17]. Thus, the criterion in Corollary 3.11 is the same as [Nikshych 2008, Corollary 3.6].

3D. Example: the relative center of a crossed product category. Let $G$ be a finite group and let $g \mapsto T_{g}, g \in G$, be an action of $G$ on a fusion category $\mathscr{D}$. Let $\mathscr{C}:=\mathscr{D} \rtimes G$ be the crossed product category defined in Section 2D. It has a natural grading

$$
\mathscr{b}=\bigoplus_{g \in G} \mathscr{C}_{g}, \quad \text { where } \mathscr{C}_{g}=\{Y \otimes g \mid Y \in \mathscr{D}\} .
$$

We describe the braided $G$-crossed fusion category structure on the relative center

$$
\mathscr{L}_{\mathscr{D}}(\mathscr{C})=\bigoplus_{g \in G} \mathscr{L}_{\mathscr{D}}\left(\mathscr{C}_{g}\right)
$$

By definition, the objects of $\mathscr{L}_{\mathscr{D}}\left(\mathscr{C}_{g}\right)$ are pairs $(Y \otimes g, \gamma)$, where $Y \in \mathscr{D}$ and

$$
\gamma=\left\{\gamma_{X}: X \otimes Y \stackrel{\sim}{\rightarrow} Y \otimes T_{g}(X)\right\}_{X \in \mathscr{D}}
$$

is a natural family of isomorphisms satisfying natural compatibility conditions. Thus, $\mathscr{L}_{\mathscr{D}}\left(\mathscr{G}_{g}\right)$ can be viewed as a "deformation" of $\mathscr{L}(\mathscr{D})$ by means of $T_{g}$.

The action of $G$ on $\mathscr{D}$ induces an action $h \mapsto \tilde{T}_{h}$ on $\mathscr{L}_{\mathscr{D}}(\mathscr{C})$ defined as follows. Applying $T_{h}, h \in G$, to $\gamma_{T^{-1}}(X)$ in (33), we obtain an isomorphism

$$
\tilde{\gamma}_{X}: X \otimes T_{h}(Y) \stackrel{\sim}{\rightarrow} T_{h}(Y) \otimes T_{h g h^{-1}}(X) .
$$

Set $\tilde{T}_{h}(Y \otimes g, \gamma):=\left(T_{h}(Y) \otimes h g h^{-1}, \tilde{\gamma}\right)$. Thus, $\tilde{T}_{h}$ maps $\mathscr{L}_{\mathscr{D}}\left(\mathscr{C}_{g}\right)$ to $\mathscr{L}_{\mathscr{D}}\left(\mathscr{C}_{h g h^{-1}}\right)$. 
Finally, the $G$-braiding between objects $(X \otimes h) \in \mathscr{E}_{\mathscr{D}}\left(\mathscr{C}_{h}\right)$ and $(Y \otimes g) \in \mathscr{L}_{\mathscr{D}}\left(\mathscr{C}_{g}\right)$ comes from the isomorphism

$$
\begin{aligned}
(X \otimes h) \otimes(Y \otimes g)=\left(X \otimes T_{h}(Y)\right) \otimes h g \stackrel{\tilde{\tilde{r}}}{\rightarrow}\left(T_{h}(Y)\right. & \left.\otimes T_{h g h^{-1}}(X)\right) \otimes h g \\
& =\left(T_{h}(Y) \otimes h g h^{-1}\right) \otimes(X \otimes h) \\
& =\tilde{T}_{h}(Y \otimes g) \otimes(X \otimes h) .
\end{aligned}
$$

By Theorem 3.5, the category $\mathscr{L}(\mathscr{D} \rtimes G) \cong \mathscr{L}\left(\mathscr{D}^{G}\right)$ is equivalent to the equivariantization of the braided $G$-crossed category above.

\section{The centers of Tambara-Yamagami categories}

Our goal in this section is to apply techniques developed in Section 3 to TambaraYamagami categories introduced in [Tambara and Yamagami 1998] (see Section 4A below for the definition). Namely, using the techniques in Section 3 we establish a criterion for a Tambara-Yamagami category to be group-theoretical. We then use this criterion together with Corollary 3.11 to produce a series of non-grouptheoretical semisimple Hopf algebras. In this section we assume that our ground field $k$ is the field of complex numbers $\mathbb{C}$. We begin by recalling the definition of a Tambara-Yamagami category.

4A. Definition of Tambara-Yamagami categories. Let $\mathbb{Z}_{2}=\left\langle\delta \mid \delta^{2}=1\right\rangle$ be the cyclic group of order 2.

Tambara and Yamagami [1998] completely classified all $\mathbb{Z}_{2}$-graded fusion categories in which all but one simple objects are invertible and the noninvertible simple object has nontrivial graded degree.

They showed that any such category $\mathscr{T} \mathscr{Y}(A, \chi, \tau)$ is determined, up to an equivalence, by a finite abelian group $A$, a nondegenerate symmetric bilinear form $\chi: A \times A \rightarrow k^{\times}$, and a square root $\tau \in k$ of $|A|^{-1}$. The category $\mathscr{T} \mathscr{Y}(A, \chi, \tau)$ is described as follows. It is a skeletal category (that is, such that any two isomorphic objects are equal) with simple objects $\{a \mid a \in A\}$ and $m$, and tensor product

$$
a \otimes b=a+b, \quad a \otimes m=m, \quad m \otimes a=m, \quad m \otimes m=\bigoplus_{a \in A} a,
$$

for all $a, b \in A$, and the unit object $0 \in A$. The associativity constraints are given by

$$
\begin{aligned}
\alpha_{a, b, c} & =\operatorname{id}_{a+b+c}, \quad \alpha_{a, b, m}=\operatorname{id}_{m}, \quad \alpha_{a, m, b}=\chi(a, b) \mathrm{id}_{m}, \quad \alpha_{m, a, b}=\mathrm{id}_{m}, \\
\alpha_{a, m, m} & =\bigoplus_{b \in A} \operatorname{id}_{b}, \quad \alpha_{m, a, m}=\bigoplus_{b \in A} \chi(a, b) \mathrm{id}_{b}, \\
\alpha_{m, m, a} & =\bigoplus_{b \in A} \operatorname{id}_{b}, \quad \alpha_{m, m, m}=\bigoplus_{a, b \in A} \tau \chi(a, b)^{-1} \mathrm{id}_{m} .
\end{aligned}
$$


The unit constraints are the identity maps. The category $\mathscr{T} \mathscr{Y}(A, \chi, \tau)$ is rigid with $a^{*}=-a$ and $m^{*}=m$ (with obvious evaluation and coevaluation maps).

Let $n:=|A|$. The dimensions of simple objects of $\mathscr{T} \mathscr{Y}(A, \chi, \tau)$ are $\operatorname{FPdim}(a)=$ $1, a \in A$, and $\operatorname{FPdim}(m)=\sqrt{n}$. We have $\operatorname{FPdim}(\mathscr{T} \mathscr{Y}(A, \chi, \tau))=2 n$.

The $\mathbb{Z}_{2}$-grading on $\mathscr{T} \mathscr{Y}(A, \chi, \tau)$ is

$$
\mathscr{T} \mathscr{Y}(A, \chi, \tau)=\mathscr{T} \mathscr{Y}(A, \chi, \tau)_{1} \oplus \mathscr{T} \mathscr{Y}(A, \chi, \tau)_{\delta},
$$

where $\mathscr{T} \mathscr{Y}(A, \chi, \tau)_{1}$ is the full fusion subcategory generated by the invertible objects $a \in A$ and $\mathscr{T} \mathscr{Y}(A, \chi, \tau)_{\delta}$ is the full abelian subcategory generated by the object $m$.

Let $\mathscr{C}:=\mathscr{T} \mathscr{Y}(A, \chi, \tau)$ and $\mathscr{D}:=\mathscr{T} \mathscr{Y}(A, \chi, \tau)_{1}$.

4B. Braided $\mathbb{Z}_{2}$-crossed category $\mathscr{L}_{\mathscr{D}}(\mathscr{C})$. First, let us describe the simple objects of $\mathscr{L}_{\mathscr{D}}(\mathscr{C})=\mathscr{L}\left(\mathscr{C}_{1}\right) \oplus \mathscr{L}_{\mathscr{D}}\left(\mathscr{C}_{\delta}\right)$. Let $\widehat{A}:=\operatorname{Hom}\left(A, k^{\times}\right)$. Clearly, $\mathscr{L}\left(\mathscr{C}_{1}\right)=\mathscr{L}\left(\operatorname{Vec}_{A}\right)$, so its simple objects are parameterized by $(a, \phi) \in A \times \widehat{A}$. The object $X_{(a, \phi)}$ corresponding to such a pair is equal to $a$ as an object of $\mathscr{C}$ and its central structure is given by

$$
\phi(x) \operatorname{id}_{a+x}: x \otimes X_{(a, \phi)} \stackrel{\sim}{\rightarrow} X_{(a, \phi)} \otimes x .
$$

Using Definition 2.1 we see that simple objects of $\mathscr{L}_{\mathscr{D}}\left(\mathscr{C}_{\delta}\right)$ are parameterized by functions $\rho: A \rightarrow k^{\times}$satisfying

$$
\rho(a+b)=\chi(a, b)^{-1} \rho(a) \rho(b), \quad a, b \in A
$$

(clearly, such functions form a torsor over $\widehat{A}$ ). The corresponding object $Z_{\rho}$ is equal to $m$ as an object of $\mathscr{b}$ and has the relative central structure

$$
\rho(x) \operatorname{id}_{m}: x \otimes Z_{\rho} \stackrel{\sim}{\rightarrow} Z_{\rho} \otimes x, \quad x \in A .
$$

Let $A \rightarrow \widehat{A}: a \mapsto \widehat{a}$ be the homomorphism defined by $\widehat{a}(x)=\chi(x, a)$. Similarly, let $\widehat{A} \rightarrow A: \phi \mapsto \widehat{\phi}$ be the homomorphism defined by $\phi(x)=\chi(x, \widehat{\phi})$ (recall that $\chi$ is nondegenerate). Clearly, these two maps are inverses of each other.

The fusion rules of $\mathscr{L}_{\mathscr{D}}(\mathscr{C})$ are computed using formula (3) :

$$
\begin{aligned}
X_{(a, \phi)} \otimes X_{(b, \psi)} & =X_{(a+b, \phi+\psi)}, \\
X_{(a, \phi)} \otimes Z_{\rho} & =Z_{\rho \phi(-\widehat{a})}, \\
Z_{\rho} \otimes X_{(a, \phi)} & =Z_{\rho \phi(-\widehat{a})}, \\
Z_{\rho^{\prime}} \otimes Z_{\rho} & =\bigoplus_{a \in A} X_{\left(a, \widehat{a} \rho^{\prime} / \bar{\rho}\right)} .
\end{aligned}
$$

We have $X_{(a, \phi)}^{*}=X_{(-a,-\phi)}$ and $Z_{\rho}^{*}=Z_{\bar{\rho}}$, where $\bar{\rho}(x)=\rho(-x), x \in A$. 
Using the construction given in Section $3 \mathrm{~A}$ we see that the action of $\mathbb{Z}_{2}$ on $\mathscr{L}_{\mathscr{D}}(\mathscr{C})$ is given by

$$
T_{1}=\operatorname{id}_{\mathscr{L}_{\mathscr{I}}(\mathscr{C})} ; \quad T_{\delta}\left(X_{(a, \phi)}\right)=X_{(-\widehat{\phi},-\widehat{a})}, \quad T_{\delta}\left(Z_{\rho}\right)=Z_{\rho} .
$$

The monoidal functor structure on $\mathbb{Z}_{2} \rightarrow \operatorname{Aut}_{\otimes}\left(\mathscr{L}_{\mathscr{D}}(\mathscr{C})\right)$ is given by the natural isomorphism $\gamma:=\gamma_{\delta, \delta}: T_{\delta} \circ T_{\delta} \stackrel{\sim}{\rightarrow} T_{1}$ defined by

$$
\gamma_{X_{(a, \phi)}}=\phi(a) \operatorname{id}_{X_{(a, \phi)}}, \quad \gamma_{Z_{\rho}}=\left(\tau \sum_{x \in A} \rho(x)^{-1}\right) \operatorname{id}_{Z_{\rho}} .
$$

The crossed braiding morphisms on $\mathscr{L}_{\mathscr{D}}(\mathscr{C})$ are given by

$$
\begin{aligned}
c_{X_{(a, \phi)}, X_{(b, \psi)}} & =\psi(a) \mathrm{id}_{a+b}: X_{(a, \phi)} \otimes X_{(b, \psi)} \stackrel{\sim}{\rightarrow} X_{(b, \psi)} \otimes X_{(a, \phi)}, \\
c_{X_{(a, \phi)}, Z_{\rho}} & =\rho(a) \operatorname{id}_{m}: X_{(a, \phi)} \otimes Z_{\rho} \stackrel{\sim}{\rightarrow} Z_{\rho} \otimes X_{(a, \phi)}, \\
c_{Z_{\rho}, X_{(a, \phi)}} & =\operatorname{id}_{m}: Z_{\rho} \otimes X_{(a, \phi)} \stackrel{\sim}{\rightarrow} X_{(-\widehat{\phi},-\widehat{a})} \otimes Z_{\rho}, \\
c_{Z_{\rho^{\prime}}, Z_{\rho}} & =\oplus_{a \in A} \rho(-a)^{-1} \mathrm{id}_{a}: Z_{\rho^{\prime}} \otimes Z_{\rho} \stackrel{\sim}{\rightarrow} Z_{\rho} \otimes Z_{\rho^{\prime}} .
\end{aligned}
$$

4C. The equivariantization category $\mathscr{L}_{\mathscr{D}}(\mathscr{C})^{\mathbb{Z}_{2}}$. A simple calculation of $\mathbb{Z}_{2}$-equivariant objects in $\mathscr{L}_{\mathscr{D}}(\mathscr{C})$ establishes the following.

Proposition 4.1. The following is a complete list of simple objects of $\mathscr{L}_{\mathscr{D}}\left(\mathscr{C}^{\mathbb{Z}_{2}} \cong\right.$ $\mathscr{L}(\mathscr{T} Y(A, \chi, \tau))$ up to an isomorphism:

(1) $2 n$ invertible objects parameterized by pairs $(a, \epsilon)$, where $a \in A$ and $\epsilon^{2}=$ $\chi(a, a)^{-1}$. The corresponding object $X_{a, \epsilon}$ is equal to $X_{(a,-\widehat{a})}$ as an object of $\mathscr{L}_{\mathscr{D}}(\mathfrak{C})$ and has $\mathbb{Z}_{2}$-equivariant structure

$$
\epsilon \operatorname{id}_{X_{(a,-\widehat{a})}}: T_{\delta}\left(X_{(a,-\widehat{a})}\right) \stackrel{\sim}{\rightarrow} X_{(a,-\widehat{a})} ;
$$

(2) $\frac{n(n-1)}{2}$ two-dimensional objects parameterized by unordered pairs $(a, b)$ of distinct objects in $A$. The corresponding object $Y_{a, b}$ is equal to $X_{(a,-\widehat{b})} \oplus$ $X_{(b,-\widehat{a})}$ as an object of $\mathscr{L}_{\mathscr{D}}(\mathscr{C})$ and has $\mathbb{Z}_{2}$-equivariant structure

$$
\left(\operatorname{id}_{X_{(a,-\widehat{b})}} \oplus \chi(a, b)^{-1} \operatorname{id}_{X_{(b,-\widehat{a})}}\right): T_{\delta}\left(X_{(a,-\widehat{b})} \oplus X_{(b,-\widehat{a})}\right) \stackrel{\sim}{\rightarrow} X_{(a,-\widehat{b})} \oplus X_{(b,-\widehat{a})} ;
$$

(3) $2 n \sqrt{n}$-dimensional objects parameterized by pairs $(\rho, \Delta)$, where $\rho: A \rightarrow k^{\times}$ satisfies (36) and $\Delta^{2}=\tau \sum_{x \in A} \rho(x)^{-1}$. The corresponding object $Z_{\rho, \Delta}$ is equal to $Z_{\rho}$ as an object of $\mathscr{L}_{\mathscr{D}}(\mathscr{C})$ and has $\mathbb{Z}_{2}$-equivariant structure

$$
\Delta \operatorname{id}_{Z_{\rho}}: T_{\delta}\left(Z_{\rho}\right) \stackrel{\sim}{\rightarrow} Z_{\rho} .
$$

Recall from [Etingof et al. 2005] that in a braided fusion category of an integer Frobenius-Perron dimension there is a canonical choice of a twist $\theta$ such that the categorical dimensions of objects coincide with their Frobenius-Perron 
dimensions. Namely, for any simple object $X$ the scalar $\theta_{X}$ is defined in such a way that the composition

$$
\mathbf{1} \stackrel{\operatorname{coev}_{X}}{\longrightarrow} X \otimes X^{*} \stackrel{\theta_{X} c_{X, X^{*}}}{\longrightarrow} X^{*} \otimes X \stackrel{\mathrm{ev}_{X}}{\longrightarrow} \mathbf{1}
$$

is equal to $\operatorname{FPdim}(X) \operatorname{id}_{X}$.

Let $\theta$ be the canonical twist on $\mathscr{L}(\mathscr{C})$. Using the previous observation, explicit formulas from Section 4B, and Section 2F, we immediately obtain the following.

$$
\theta_{X_{a, \epsilon}}=\chi(a, a)^{-1}, \quad \theta_{Y_{a, b}}=\chi(a, b)^{-1}, \quad \theta_{Z_{\rho, \Delta}}=\Delta .
$$

Using the fusion rules of $\mathscr{L}(\mathscr{C})$ (which may be computed using the explicit formulas in Section 4B), values of the twists above, and the well known formula

$$
S_{X, Y}=\theta_{X}^{-1} \theta_{Y}^{-1} \sum_{Z} N_{X, Y}^{Z} \theta_{Z} d_{Z}
$$

we obtain the $S$ - and $T$-matrices of $\mathscr{E}(\mathscr{C})$ :

$$
\begin{aligned}
S_{X_{a, \epsilon}, X_{a^{\prime}, \epsilon^{\prime}}} & =\chi\left(a, a^{\prime}\right)^{2}, \quad S_{X_{a, \epsilon}, Y_{b, c}}=2 \chi(a, b+c), \\
S_{X_{a, \epsilon}, Z_{\rho, \Delta}} & =\epsilon \sqrt{n} \rho(a), \quad S_{Y_{a, b}, Y_{c, d}}=2(\chi(a, d) \chi(b, c)+\chi(a, c) \chi(b, d)), \\
S_{Y_{a, b}, Z_{\rho, \Delta}} & =0, \quad S_{Z_{\rho, \Delta}, Z_{\rho^{\prime}, \Delta^{\prime}}}=\frac{1}{\Delta \Delta^{\prime}} \sum_{a \in A} \chi(a, a)^{2} \rho(a) \rho^{\prime}(a) ; \\
T_{X_{a, \epsilon}} & =\chi(a, a)^{-1}, \quad T_{Y_{a, b}}=\chi(a, b)^{-1}, \quad T_{Z_{\rho, \Delta}}=\Delta .
\end{aligned}
$$

Proposition 4.2. The maximal pointed subcategory of $\mathscr{L}(\mathscr{C})$ is nondegenerate if and only if $|A|$ is odd.

Proof. Let $a \in A$ be an element of order 2. Then $X_{a, \epsilon}$ centralizes every invertible object of $\mathscr{L}(\mathscr{C})$.

Remark 4.3. We note that simple objects and the $S$ - and $T$-matrices of $\mathscr{L}(\mathscr{C})$ were described in [Izumi 2001] using very different methods.

4D. A criterion for a Tambara-Yamagami category to be group-theoretical. The group $A \times \widehat{A}$ is equipped with a canonical nondegenerate quadratic form $q: A \times \widehat{A} \rightarrow$ $k^{\times}$given by

$$
q((a, \phi)):=\phi(a), \quad(a, \phi) \in A \times \widehat{A} .
$$

We will call a subgroup $B \subset A \times \widehat{A}$ Lagrangian if $\left.q\right|_{B}=1$ and $B=B^{\perp}$ with respect to the bilinear form defined by $q$. Lagrangian subgroups of $A \times \widehat{A}$ correspond to Lagrangian subcategories of $\mathscr{E}\left(\operatorname{Vec}_{A}\right) \cong \operatorname{Vec}_{A \times \widehat{A}}$.

The braided tensor autoequivalence $T_{\delta}$ of $\mathscr{L}\left(\operatorname{Vec}_{A}\right)$ defined in Section $4 \mathrm{~B}$ determines an order 2 automorphism of $A \times \widehat{A}$, which we denote simply by $\delta$ :

$$
\delta((a, \phi))=(-\widehat{\phi},-\widehat{a}), \quad(a, \phi) \in A \times \widehat{A} .
$$


Definition 4.4. We will say that a subgroup $L \subset A$ is Lagrangian (with respect to $\chi$ ) if $L=L^{\perp}$ with respect to the inner product on $A$ given by $\chi$. Equivalently, $|L|^{2}=|A|$ and $\left.\chi\right|_{L}=1$.

Lemma 4.5. Let $A$ be an abelian 2-group such that $|A|=2^{2 n}$ and let $\chi$ be a nondegenerate symmetric bilinear form on A. Then A contains a Lagrangian subgroup.

Proof. It suffices to show that $A$ contains an isotropic element, that is, an element $x \in A, x \neq 0$, such that $\chi(x, x)=1$. Then one can pass from $A$ to $\langle x\rangle^{\perp} /\langle x\rangle$ and use induction.

Suppose that $A$ is cyclic with a generator $a$. Then $2^{2 n} a=0$ and $\chi(a, a)$ is a $\left(2^{2 n}\right)$ th root of unity, hence $\chi\left(2^{n} a, 2^{n} a\right)=\chi(a, a)^{2^{2 n}}=1$.

If $A$ is not cyclic then it contains a subgroup $A_{0}=\mathbb{Z} / 2 \mathbb{Z} \oplus \mathbb{Z} / 2 \mathbb{Z}$. Let $x_{1}, x_{2}$ be distinct nonzero elements of $A_{0}$. Suppose $\chi\left(x_{i}, x_{i}\right) \neq 1, i=1,2$. Then $\chi\left(x_{i}, x_{i}\right)=$ -1 and $\chi\left(x_{1}+x_{2}, x_{1}+x_{2}\right)=1$, as desired.

Theorem 4.6. Let $\mathscr{C}=\mathscr{T} \mathscr{Y}(A, \chi, \tau)$ be a Tambara-Yamagami fusion category. Then $\mathscr{C}$ is group-theoretical if and only if A contains a Lagrangian subgroup (with respect to $\chi$ ).

Proof. By Corollary 3.10, $\mathscr{C}$ is group-theoretical if and only if $\mathscr{L}(\mathscr{D})$ contains a $T_{\delta}$-stable Lagrangian subcategory. Equivalently, $\mathscr{C}$ is group-theoretical if and only if $A \times \widehat{A}$ contains a Lagrangian subgroup $B$ stable under the action

$$
(a, \phi) \mapsto(\widehat{\phi}, \widehat{a}) \text {. }
$$

This condition on $B$ is the same as being stable under the action of $\delta$ from (41).

Let $L$ be a Lagrangian (with respect to $\chi$ ) subgroup of $A$ and let $\widehat{L}:=\{\widehat{a} \mid a \in L\}$. Then $L \times \widehat{L}$ is a Lagrangian subgroup of $A \times \widehat{A}$ stable under (42). Hence $\mathscr{C}$ is grouptheoretical.

Conversely, suppose that $\mathscr{C}$ is group-theoretical. Let us write $A=A_{\text {even }} \oplus A_{\text {odd }}$, where $A_{\text {even }}$ is the Sylow 2-subgroup of $A$ and $A_{\text {odd }}$ is the maximal odd order subgroup of $A$. Since $|A|$ must be a square, we conclude that $\left|A_{\text {even }}\right|$ is a square, and so $A_{\text {even }}$ contains a Lagrangian subgroup with respect to $\left.\chi\right|_{A_{\text {even }}}$ by Lemma 4.5.

So it remains to show that $A_{\text {odd }}$ contains a Lagrangian subgroup with respect to $\left.\chi\right|_{A_{\text {odd }}}$. For this end we may assume that $|A|$ is odd. Let $B \subset A \times \widehat{A}$ be a Lagrangian subgroup stable under (42). Then $B=B_{+} \oplus B_{-}$, where

$$
B_{ \pm}:=\{(a, \pm \widehat{a}) \mid(a, \pm \widehat{a}) \in B\} .
$$

Let $L_{ \pm}=B_{ \pm} \cap(A \times\{1\})$. Then $\left|L_{+}\right|\left|L_{-}\right|=|A|$, and $\left.\chi\right|_{L_{ \pm}}=1$. Hence, $L_{ \pm}$are Lagrangian subgroups of $A$.

Remark 4.7. It was observed in [Etingof et al. 2005, Remark 8.48] that for an odd prime $p$ and elliptic bicharacter $\chi$ on $A=(\mathbb{Z} / p \mathbb{Z})^{2}$, the category $\mathscr{T} \mathscr{Y}\left((\mathbb{Z} / p \mathbb{Z})^{2}, \chi, \tau\right)$ is not group-theoretical. The criterion from Theorem 4.6 extends this observation. 
4E. A series of non-group-theoretical semisimple Hopf algebras obtained from Tambara-Yamagami categories. Here we apply Corollary 3.11 to produce a series of non-group-theoretical fusion categories admitting fiber functors (that is, representation categories of non-group-theoretical semisimple Hopf algebras), generalizing examples constructed in [Nikshych 2008]. We refer the reader to [Montgomery 1993] as a reference on Hopf algebra theory.

Let $A$ be a finite abelian group with a nondegenerate bilinear form $\chi$. Let $\operatorname{Aut}(A, \chi)$ denote the group of automorphisms of $A$ preserving $\chi$.

The following proposition was proved in [Nikshych 2008, Proposition 2.10].

Proposition 4.8. There is an action of $\operatorname{Aut}(A, \chi)$ on $\mathscr{T} \mathscr{Y}(A, \chi, \tau)$ given by $g \mapsto$ $T_{g}$, where

$$
T_{g}(A)=g(a), \quad T_{g}(m)=m, \quad a \in A, g \in \operatorname{Aut}(A, \chi),
$$

with the tensor structure of $T_{g}$ given by identity morphisms.

Corollary 4.9. Let $G$ be a subgroup of $\operatorname{Aut}(A, \chi)$. Then the fusion category $\mathscr{T} \mathscr{Y}(A, \chi, \tau)^{G}$ is group-theoretical if and only if there is a Lagrangian subgroup of $(A, \chi)$ stable under the action of $G$.

Proof. Combine Corollary 3.11 and Theorem 4.6.

We will say that a nondegenerate symmetric bilinear form $\chi: A \times A \rightarrow k^{\times}$is hyperbolic if there are Lagrangian subgroups $L, L^{\prime} \subset A$ such that $A=L \oplus L^{\prime}$. In this case $L^{\prime}$ is isomorphic to the group $\widehat{L}=\operatorname{Hom}\left(L, k^{\times}\right)$of characters of $L$ and $\chi$ is identified with the canonical bilinear form on $L \oplus \widehat{L}$.

It was demonstrated in Tambara [2000] that when $n=|A|$ is odd the category $\mathscr{T} \mathscr{Y}(A, \chi, \tau)$ admits a fiber functor (that is, $\mathscr{T} \mathscr{Y}(A, \chi, \tau)$ is equivalent to the representation category of a semisimple Hopf algebra) if and only if $\tau^{-1}$ is a positive integer and $\chi$ is hyperbolic.

Corollary 4.10. Let $p$ be an odd prime, let $L=(\mathbb{Z} / p \mathbb{Z})^{N}, N \geq 1$, let $A=L \oplus \widehat{L}$, and let $\chi: A \times A \rightarrow k^{\times}$be the canonical bilinear form defined by

$$
\chi((a, \phi),(b, \psi))=\psi(a) \phi(b), \quad a, b \in A, \phi, \psi \in \widehat{A} .
$$

Suppose that $G$ is a subgroup of $\operatorname{Aut}(A, \chi)$ not contained in any conjugate of $\operatorname{Aut}(L) \subset \operatorname{Aut}(A, \chi)$. Then the equivariantization category $\mathscr{T} \mathscr{Y}\left(A, \chi, p^{-N}\right)^{G}$ is a non-group-theoretical fusion category equivalent to the representation category of a semisimple Hopf algebra of dimension $2 p^{2 N}|G|$.

Proof. Note that $\operatorname{Aut}(A, \chi)$ acts transitively on the set of Lagrangian subgroups of $(A, \chi)$ and the stabilizer of $L$ is $\operatorname{Aut}(L)$. Apply Corollary 4.9. 
Remark 4.11. The series of fusion categories in Corollary 4.10 extends the one constructed in [Nikshych 2008], where the case of $N=1$ and $G=\mathbb{Z} / 2 \mathbb{Z}$ was considered.

\section{Examples of modular categories arising from quadratic forms}

As before, let $\mathscr{C}:=\mathscr{T} \mathscr{Y}(A, \chi, \tau)$ be a Tambara-Yamagami category and let $\mathscr{D}:=$ $\mathscr{T} \mathscr{Y}(A, \chi, \tau)_{1}$ be the trivial component of $\mathbb{Z}_{2}$-grading of $\mathscr{T} \mathscr{Y}(A, \chi, \tau)$. In this section we assume that our ground field $k$ is the field of complex numbers $\mathbb{C}$.

Suppose that the symmetric bicharacter $\chi: A \times A \rightarrow k^{\times}$comes from a quadratic form on $A$, that is, there is a function $q: A \rightarrow k^{\times}$such that

$$
q(a+b)=q(a) q(b) \chi(a, b), \quad a, b \in A \quad \text { and } \quad q(-a)=q(a) .
$$

From the description obtained in Section 4B we observe that $\mathscr{L}_{\mathscr{D}}(\mathscr{C})$ contains a fusion subcategory spanned by the simple objects $X_{(a, \widehat{a})}, a \in A$, and $Z_{q^{-1}}$. It is clear from the Tambara-Yamagami classification in Section 4A that this category is equivalent to $\mathscr{b}$.

Proposition 5.1. Suppose that the symmetric bicharacter $\chi$ comes from a quadratic form on $A$. Then $\mathscr{b}$ admits a $\mathbb{Z}_{2}$-crossed braided category structure. The equivariantization $\mathscr{C}^{\mathbb{Z}_{2}}$ is nondegenerate if and only if $|A|$ is odd.

Proof. Clearly, $\mathscr{C}$ inherits the $\mathbb{Z}_{2}$-crossed braided category structure from $\mathscr{L}_{\mathscr{D}}(\mathscr{C})$. The nondegeneracy claim follows from Proposition 4.2 and Remark 2.13.

Let us assume that $n:=|A|$ is odd. Then $\chi$ corresponds to a unique quadratic form $q$. Let $\mathscr{E}(q, \pm):=\mathscr{C}^{\mathbb{Z}_{2}}$ be the modular category constructed in Proposition 5.1 (the \pm corresponding to $\tau= \pm \frac{1}{\sqrt{n}}$, respectively). In what follows we describe the fusion rules and $S$ - and $T$-matrices of $\mathscr{E}(q, \pm)$.

5A. Fusion rules of $\mathscr{E}$. Clearly, $\mathscr{E}(q, \pm)$ is a fusion category of dimension $4 n$. It has the following simple objects:

two invertible objects, $\mathbf{1}=X_{+}$and $X_{-}$;

$\frac{n-1}{2}$ two-dimensional objects $Y_{a}, a \in A-\{0\}$ (with $Y_{-a}=Y_{a}$ ); and two $\sqrt{n}$-dimensional objects $Z_{l}, l \in \mathbb{Z} / 2 \mathbb{Z}$.

Here we simplify the notation used in Section $4 \mathrm{C}$ and define

$$
X_{ \pm}:=X_{0, \pm 1}, \quad Y_{a}:=Y_{a,-a}, \quad Z_{l}:=Z_{q^{-1}, \Delta_{l}},
$$

where $\Delta_{l}, l \in \mathbb{Z} / 2 \mathbb{Z}$, are distinct square roots of $\pm \frac{1}{\sqrt{n}} \sum_{a \in A} q(a)$. 
The fusion rules of $\mathscr{E}(q, \pm)$ are given by

$$
\begin{aligned}
& X_{-} \otimes X_{-}=X_{+}, \quad X_{ \pm} \otimes Y_{a}=Y_{a}, \quad X_{+} \otimes Z_{l}=Z_{l}, \\
& X_{-} \otimes Z_{l}=Z_{l+1}, \quad Y_{a} \otimes Y_{b}=Y_{a+b} \oplus Y_{a-b}, \quad Y_{a} \otimes Y_{a}=X_{+} \oplus X_{-} \oplus Y_{2 a}, \\
& Y_{a} \otimes Z_{l}=Z_{0} \oplus Z_{1}, \quad Z_{l} \otimes Z_{l}=X_{+} \oplus\left(\oplus Y_{a}\right), Z_{l} \otimes Z_{l+1}=X_{-} \oplus\left(\oplus Y_{a}\right),
\end{aligned}
$$

where $a, b \in A(a \neq b)$ and $l \in \mathbb{Z} / 2 \mathbb{Z}$. All objects of $\mathscr{E}(q, \pm)$ are self-dual.

Remark 5.2. Note that the fusion rules of $\mathscr{E}(q, \pm)$ do not depend on the quadratic form $q$ and the number $\tau$. We show below that the $S$ - and $T$-matrices of $\mathscr{E}(q, \pm)$ do depend on $q$ and $\tau$.

\section{B. $S$ - and $T$-matrices of $\mathscr{E}$.}

Lemma 5.3. The Gauss sums corresponding to $q$ and $q^{2}$ are equal up to a sign, that is,

$$
\frac{\sum_{a \in A} q(a)^{2}}{\sum_{a \in A} q(a)} \in\{ \pm 1\} .
$$

Proof. Consider the group $A \times A$ with a nondegenerate quadratic form $Q=q \times q$. The Gaussian sum for this form is

$$
\tau(A \times A, Q)=\sum_{a, b \in A} q(a) q(b)=\tau(A, q)^{2} .
$$

The restriction of $Q$ on the diagonal subgroup $D:=\{(a, a) \mid a \in A\}$ is nondegenerate since $|A|$ is odd. The restriction of $Q$ on the orthogonal complement $D^{\perp}=\{(a,-a) \mid a \in A\}$ is nondegenerate as well. By the multiplicativity of Gaussian sums we have

$$
\tau(A \times A, Q)=\tau(D, Q) \tau\left(D^{\perp}, Q\right)=\left(\sum_{a \in A} q(a)^{2}\right)^{2},
$$

which implies the result.

Using the formulas for the $S$ - and $T$ - matrices of $\mathscr{E}(\mathscr{C})$ given in Section $4 \mathrm{C}$ we can write down the $S$ - and $T$ - matrices of $\mathscr{E}(q, \pm)$ :

$$
\begin{aligned}
S_{X_{ \pm}, X_{ \pm}} & =1, \quad S_{X_{\mp}, X_{ \pm}}=1, \quad S_{X_{ \pm}, Y_{a}}=2, \quad S_{Y_{a}, Z_{l}}=0, \\
S_{X_{+}, Z_{l}} & =\sqrt{n}, \quad S_{X_{-}, Z_{l}}=-\sqrt{n}, \quad S_{Y_{a}, Y_{b}}=2\left(\frac{q(a+b)^{2}}{q(a)^{2} q(b)^{2}}+\frac{q(a)^{2} q(b)^{2}}{q(a+b)^{2}}\right), \\
S_{Z_{l}, Z_{l}} & =\left\{\begin{array}{l} 
\pm \sqrt{n} \text { if the Gauss sums of } q \text { and } q^{2} \text { coincide, } \\
\mp \sqrt{n} \text { otherwise, }
\end{array}\right. \\
S_{Z_{l}, Z_{l+1}} & =\left\{\begin{array}{l}
\mp \sqrt{n} \text { if the Gauss sums of } q \text { and } q^{2} \text { coincide, } \\
\pm \sqrt{n} \text { otherwise. }
\end{array}\right.
\end{aligned}
$$




$$
T_{X_{ \pm}}=1, \quad T_{Y_{a}}=q(a)^{2}, \quad T_{Z_{l}}=\Delta_{l} .
$$

(Recall that $\Delta_{l}, l \in \mathbb{Z} / 2 \mathbb{Z}$, are distinct square roots of $\pm \frac{1}{\sqrt{n}} \sum_{a \in A} q(a)$.)

5C. Example with $A=\mathbb{Z} / \boldsymbol{p} \mathbb{Z} \times \mathbb{Z} / \boldsymbol{p} \mathbb{Z}$. Let $p$ be an odd prime and let $A:=$ $\mathbb{Z} / p \mathbb{Z} \times \mathbb{Z} / p \mathbb{Z}$. Let $\left(\frac{\dot{p}}{p}\right)$ denote the Legendre symbol modulo $p$, that is, $\left(\frac{a}{p}\right)=1$ if $a \in(\mathbb{Z} / p \mathbb{Z})^{\times}$is a square modulo $p$ and -1 otherwise.

Let $a, b \in(\mathbb{Z} / p \mathbb{Z})^{\times}$and $\xi:=e^{2 \pi i / p}$. Consider the following nondegenerate quadratic form $q$ on $A$ :

$$
q\left(x_{1}, x_{2}\right)=\xi^{a x_{1}^{2}-b x_{2}^{2}} .
$$

It is hyperbolic if $\left(\frac{a b}{p}\right)=1$ and elliptic if $\left(\frac{a b}{p}\right)=-1$.

Lemma 5.4. For every $a, b \in A^{\times}$, we have

$$
\sum_{x \in \mathbb{Z} / p \mathbb{Z}} \xi^{a x^{2}}= \begin{cases}\left(\frac{a}{p}\right) \sqrt{p} & \text { if } p \equiv 1(\bmod 4), \\ \left(\frac{a}{p}\right) i \sqrt{p} & \text { if } p \equiv 3(\bmod 4),\end{cases}
$$

and

$$
\sum_{\left(x_{1}, x_{2}\right) \in \mathbb{Z} / p \mathbb{Z} \times \mathbb{Z} / p \mathbb{Z}} \xi^{a x_{1}^{2}-b x_{2}^{2}}=\left(\frac{a b}{p}\right) p .
$$

Proof. The first assertion is well known; see, for example, [Ireland and Rosen 1990]. The second assertion is an easy consequence of the first.

Using Lemma 5.4 we can explicitly write the $S$-matrix of $\mathscr{E}(q, \pm)$ :

$$
\begin{aligned}
& S_{X_{ \pm}, X_{ \pm}}=1, S_{X_{\mp}, X_{ \pm}}=1, \quad S_{X_{ \pm}, Y_{\left(x_{1}, x_{2}\right)}}=2, \\
& S_{X_{+}, Z_{l}}=p, S_{X_{-}, Z_{l}}=-p, \quad S_{Y_{\left(x_{1}, x_{2}\right)}, Y_{\left(y_{1}, y_{2}\right)}}=4 \operatorname{Re}\left(\xi^{4 a x_{1} y_{1}-4 b x_{2} y_{2}}\right), \\
& S_{Y_{\left(x_{1}, x_{2}\right)}, Z_{l}}=0, \quad S_{Z_{l}, Z_{l}}= \pm p, \quad S_{Z_{l}, Z_{l+1}}=\mp p,
\end{aligned}
$$

and its $T$-matrix:

$$
T_{X_{ \pm}}=1, \quad T_{Y_{\left(x_{1}, x_{2}\right)}}=\xi^{2 a x_{1}^{2}-2 b x_{2}^{2}}, \quad T_{Z_{l}}=\Delta_{l},
$$

where $\Delta_{l}, l \in \mathbb{Z} / 2 \mathbb{Z}$, are distinct square roots of $\pm\left(\frac{a b}{p}\right)$.

The central charge of the modular category $\mathscr{E}(q, \pm)$ is

$$
\zeta(\mathscr{E}(q, \pm))=\left(\frac{a b}{p}\right) .
$$

Below we give the $S$ - and $T$-matrices of the modular category $\mathscr{E}(q, \pm)$ for $p=$ 3. Order simple objects of $\mathscr{E}(q, \pm)$ as follows: $1, X_{-}, Y_{(0,1)}, Y_{(1,0)}, Y_{(1,1)}, Y_{(1,2)}$, $Z_{+}, Z_{-}$. There are four modular categories $\mathscr{E}(q, \pm)$ of dimension 36 corresponding to the choices of hyperbolic/elliptic $q$ and $\tau= \pm \frac{1}{3}$. 
(a) When $q$ is hyperbolic we have

$$
\begin{aligned}
S & =\left(\begin{array}{cccccccc}
1 & 1 & 2 & 2 & 2 & 2 & 3 & 3 \\
1 & 1 & 2 & 2 & 2 & 2 & -3 & 3 \\
2 & 2 & -2 & 4 & -2 & -2 & 0 & 0 \\
2 & 2 & 4 & -2 & -2 & -2 & 0 & 0 \\
2 & 2 & -2 & -2 & 4 & -2 & 0 & 0 \\
2 & 2 & -2 & -2 & -2 & 4 & 0 & 0 \\
3 & -3 & 0 & 0 & 0 & 0 & \pm 3 & \mp 3 \\
3 & -3 & 0 & 0 & 0 & 0 & \mp 3 & \pm 3
\end{array}\right) \\
T & =\operatorname{diag}\left\{1,1, \xi^{2}, \xi, 1,1,1,-1\right\} \quad \text { when } \tau=\frac{1}{3}, \\
T & =\operatorname{diag}\left\{1,1, \xi^{2}, \xi, 1,1, i,-i\right\} \quad \text { when } \tau=-\frac{1}{3} .
\end{aligned}
$$

Note that both the corresponding modular categories are group-theoretical with central charge 1 ; in fact the one with $\tau=\frac{1}{3}$ is equivalent to the representation category of the double $D\left(S_{3}\right)$ of the symmetric group $S_{3}$ and the one with $\tau=-\frac{1}{3}$ is equivalent to the twisted double of $S_{3}$.

(b) When $q$ is elliptic we have

$$
\begin{aligned}
S= & \left(\begin{array}{cccccccc}
1 & 1 & 2 & 2 & 2 & 2 & 3 & 3 \\
1 & 1 & 2 & 2 & 2 & 2 & -3 & 3 \\
2 & 2 & -2 & 4 & -2 & -2 & 0 & 0 \\
2 & 2 & 4 & -2 & -2 & -2 & 0 & 0 \\
2 & 2 & -2 & -2 & -2 & 4 & 0 & 0 \\
2 & 2 & -2 & -2 & 4 & -2 & 0 & 0 \\
3 & -3 & 0 & 0 & 0 & 0 & \pm 3 & \mp 3 \\
3 & -3 & 0 & 0 & 0 & 0 & \mp 3 & \pm 3
\end{array}\right), \\
T & =\operatorname{diag}\left\{1,1, \xi, \xi, \xi^{2}, \xi^{2}, i,-i\right\} \quad \text { when } \tau=\frac{1}{3}, \\
T & =\operatorname{diag}\left\{1,1, \xi, \xi, \xi^{2}, \xi^{2}, 1,-1\right\} \quad \text { when } \tau=-\frac{1}{3} .
\end{aligned}
$$

Both the corresponding modular categories are not group-theoretical. They both have central charge -1 and so are not equivalent to centers of fusion categories. In particular, they are not equivalent to representation categories of any twisted group doubles.

5D. Example with $\boldsymbol{A}=\mathbb{Z} / \boldsymbol{p} \mathbb{Z}$. Let $p$ be an odd prime and let $A:=\mathbb{Z} / p \mathbb{Z}$. Let $a \in(\mathbb{Z} / p \mathbb{Z})^{\times}$and $\xi:=e^{2 \pi i / p}$. Up to isomorphism there are two nondegenerate quadratic forms $q$ on $A$ :

$$
q(x)=\xi^{a x^{2}}
$$


one corresponding to $\left(\frac{a}{p}\right)=1$ and another to $\left(\frac{a}{p}\right)=-1$.

Using Lemma 5.4 we can explicitly write the $S$-matrix of $\mathscr{E}(q, \pm)$ :

$$
\begin{aligned}
& S_{X_{ \pm}, X_{ \pm}}=1, \quad S_{X_{\mp}, X_{ \pm}}=1, \quad S_{X_{ \pm}, Y_{x}}=2, \\
& S_{X_{+}, Z_{l}}=\sqrt{p}, \quad S_{X_{-}, Z_{l}}=-\sqrt{p}, \quad S_{Y_{x}, Y_{y}}=4 \operatorname{Re}\left(\xi^{4 a x y}\right), \\
& S_{Y_{a}, Z_{l}}=0, \quad S_{Z_{l}, Z_{l}}= \pm\left(\frac{2}{p}\right) \sqrt{p}, \quad S_{Z_{l}, Z_{l+1}}=\mp\left(\frac{2}{p}\right) \sqrt{p} .
\end{aligned}
$$

Further, we have

$$
T_{X_{ \pm}}=1, \quad T_{Y_{x}}=\xi^{-2 a x^{2}}, \quad T_{Z_{l}}=\Delta_{l},
$$

where

$$
\Delta_{l}, l \in \mathbb{Z} / 2 \mathbb{Z}, \text { are distinct square roots of } \begin{cases} \pm\left(\frac{a}{p}\right) & \text { if } p \equiv 1(\bmod 4), \\ \pm\left(\frac{a}{p}\right) i & \text { if } p \equiv 3(\bmod 4)\end{cases}
$$

The central charge of the modular category $\mathscr{E}(q, \pm)$ is

$$
\zeta(\mathscr{E}(q, \pm))= \begin{cases}\left(\frac{2 a}{p}\right) & \text { if } p \equiv 1(\bmod 4) \\ -\left(\frac{2 a}{p}\right) i & \text { if } p \equiv 3(\bmod 4)\end{cases}
$$

Below we give the $S$ - and $T$-matrices of the modular category $\mathscr{E}(q, \pm)$ for $p=$ 3 and 5. For $p=3$ we order the simple objects as $1, X_{-}, Y_{1}, Z_{0}, Z_{1}$ and for $p=5$ we order them as $\mathbf{1}, X_{-}, Y_{1}, Y_{2}, Z_{0}, Z_{1}$. (In (c) and (d) below, $\xi=e^{2 \pi i / 5}$.)

(a) When $p=3$ and $a=1$ we have

$$
\begin{aligned}
& S=\left(\begin{array}{ccccc}
1 & 1 & 2 & \sqrt{3} & \sqrt{3} \\
1 & 1 & 2 & -\sqrt{3} & -\sqrt{3} \\
2 & 2 & -2 & 0 & 0 \\
\sqrt{3} & -\sqrt{3} & 0 & \mp \sqrt{3} & \pm \sqrt{3} \\
\sqrt{3} & -\sqrt{3} & 0 & \pm \sqrt{3} & \mp \sqrt{3}
\end{array}\right), \\
& T=\operatorname{diag}\left\{1,1, \frac{-1+i \sqrt{3}}{2}, \frac{1+i}{\sqrt{2}}, \frac{-1-i}{\sqrt{2}}\right\} \quad \text { when } \tau=\frac{1}{\sqrt{3}}, \\
& T=\operatorname{diag}\left\{1,1, \frac{-1+i \sqrt{3}}{2}, \frac{1-i}{\sqrt{2}}, \frac{-1+i}{\sqrt{2}}\right\} \quad \text { when } \tau=-\frac{1}{\sqrt{3}} .
\end{aligned}
$$

The central charge of both the corresponding modular categories is $i$. 
(b) When $p=3$ and $a=2$ we have

$$
\begin{aligned}
& S=\text { the } S \text {-matrix in (a), } \\
& T=\operatorname{diag}\left\{1,1, \frac{-1-i \sqrt{3}}{2}, \frac{1-i}{\sqrt{2}}, \frac{-1+i}{\sqrt{2}}\right\} \quad \text { when } \tau=\frac{1}{\sqrt{3}}, \\
& T=\operatorname{diag}\left\{1,1, \frac{-1-i \sqrt{3}}{2}, \frac{1+i}{\sqrt{2}}, \frac{-1-i}{\sqrt{2}}\right\} \quad \text { when } \tau=\frac{1}{\sqrt{3}} .
\end{aligned}
$$

The central charge of both the corresponding modular categories is $-i$.

(c) When $p=5$ and $a=1$ we have

$$
\begin{aligned}
& S=\left(\begin{array}{cccccc}
1 & 1 & 2 & 2 & \sqrt{5} & \sqrt{5} \\
1 & 1 & 2 & 2 & -\sqrt{5} & -\sqrt{5} \\
2 & 2 & \sqrt{5}-1 & -\sqrt{5}-1 & 0 & 0 \\
2 & 2 & -\sqrt{5}-1 & \sqrt{5}-1 & 0 & 0 \\
\sqrt{5} & -\sqrt{5} & 0 & 0 & \mp \sqrt{5} & \pm \sqrt{5} \\
\sqrt{5} & -\sqrt{5} & 0 & 0 & \pm \sqrt{5} & \mp \sqrt{5}
\end{array}\right), \\
& T=\operatorname{diag}\left\{1,1, \xi^{3}, \xi^{2}, 1,-1\right\} \quad \text { when } \tau=\frac{1}{\sqrt{5}}, \\
& T=\operatorname{diag}\left\{1,1, \xi^{3}, \xi^{2}, i,-i\right\} \quad \text { when } \tau=-\frac{1}{\sqrt{5}} \text {. }
\end{aligned}
$$

The central charge of both the corresponding modular categories is -1 .

(d) When $p=5$ and $a=2$ we have

$$
\begin{aligned}
& S=\left(\begin{array}{cccccc}
1 & 1 & 2 & 2 & \sqrt{5} & \sqrt{5} \\
1 & 1 & 2 & 2 & -\sqrt{5} & -\sqrt{5} \\
2 & 2 & -\sqrt{5}-1 & \sqrt{5}-1 & 0 & 0 \\
2 & 2 & \sqrt{5}-1 & -\sqrt{5}-1 & 0 & 0 \\
\sqrt{5} & -\sqrt{5} & 0 & 0 & \mp \sqrt{5} & \pm \sqrt{5} \\
\sqrt{5} & -\sqrt{5} & 0 & 0 & \pm \sqrt{5} & \mp \sqrt{5}
\end{array}\right), \\
& T=\operatorname{diag}\left\{1,1, \xi, \xi^{4}, i,-i\right\} \quad \text { when } \tau=\frac{1}{\sqrt{5}}, \\
& T=\operatorname{diag}\left\{1,1, \xi, \xi^{4}, 1,-1\right\} \quad \text { when } \tau=-\frac{1}{\sqrt{5}} \text {. }
\end{aligned}
$$

The central charge of both the corresponding modular categories is 1 . 


\section{Appendix: Zeroes in $S$-matrices}

There is a classical result of Burnside in character theory saying that if $\chi$ is an irreducible character of a finite group $G$ and $\chi(1)>1$, then $\chi(g)=0$ for some $g \in G$; see [Berkovich and Zhmud' 1999, Chapter 21].

In this appendix we establish a categorical analogue of this result for weakly integral modular categories. Recall from [Etingof et al. 2008] that a fusion category $\mathscr{C}$ is called weakly integral if its Frobenius-Perron dimension is an integer. In this case the Frobenius-Perron dimension of every simple object of $\mathscr{C}$ is the square root of an integer [Etingof et al. 2005].

Let $\mathscr{C}$ be a weakly integral modular category with the $S$-matrix $S$. Let $\mathcal{O}(\mathscr{C})$ denote the set of all (representatives of isomorphism classes of) simple objects of $\mathscr{C}$. Given $X \in \mathcal{O}(\mathscr{C})$ define the sets

$$
T_{X}=\left\{Y \in \mathscr{O}(\mathscr{C}) \mid S_{X, Y}=0\right\}, \quad D_{X}=\mathscr{O}(\mathscr{C})-\left(T_{X} \cup\{\mathbf{1}\}\right) .
$$

Clearly, we have a partition $\mathscr{O}(\mathscr{C})=T_{X} \cup D_{X} \cup\{\mathbf{1}\}$. Let $\mathscr{T}_{X}$ and $\mathscr{D}_{X}$ be full abelian subcategories of $\mathscr{C}$ generated by $T_{X}$ and $D_{X}$, respectively.

Let $K$ be the field extension of $\mathbb{Q}$ generated by the entries of $S$. It is known [de Boer and Goeree 1991; Coste and Gannon 1994] that there is a root of unity $\xi$ such that $K \subset \mathbb{Q}(\xi)$. In particular, the operation of taking the square of an absolute value of an element of $S$ is well defined. Let $G:=\operatorname{Gal}(K / \mathbb{Q})$. Every element $\sigma \in G$ comes from a permutation $\sigma$ of $\mathcal{O}(\mathscr{C})$ such that $\sigma\left(S_{X, Y}\right)=S_{X, \sigma(Y)}$ for all $X, Y \in \mathbb{O}(\mathscr{C})$.

Let $\mathscr{C}$ be a weakly integral modular category. It was shown in [Etingof et al. 2005] that there is a canonical spherical structure on $\mathscr{C}$ such that categorical dimensions in $\mathscr{C}$ coincide with Frobenius-Perron dimensions. Let us fix this structure for the remainder of this section. For any $X \in \mathbb{O}(\mathscr{C})$ let $d_{X}$ denote the dimension of $X$. For any full abelian subcategory $\mathscr{A}$ of $\mathscr{C}$ let $\operatorname{dim} \mathscr{A}$ denote the sum of squares of dimensions of simple objects of $\mathscr{A}$.

Theorem 6.1. Let $\mathscr{C}$ be a weakly integral modular category with the $S$-matrix $S$. Then $T_{X}$ is not empty for every noninvertible simple object $X$ of $\mathscr{C}$. That is, every row (column) of $S$ corresponding to a noninvertible simple object contains at least one zero entry.

Proof. Note that the statement of Proposition does not depend on the choice of spherical structure.

We have $\sum_{Y \in \mathcal{O}(\mathscr{C})}\left|S_{X, Y}\right|^{2}=\operatorname{dim} \mathscr{C}$; hence,

$$
1=\frac{\operatorname{dim} \mathscr{C}}{d_{X}^{2}}-\sum_{Y \in D_{X}}\left|\frac{S_{X, Y}}{d_{X}}\right|^{2}=\frac{1+\operatorname{dim} \mathscr{T}_{X}}{d_{X}^{2}}-\left(\sum_{Y \in D_{X}}\left|\frac{S_{X, Y}}{d_{X}}\right|^{2}-\frac{\operatorname{dim} \mathscr{D}_{X}}{d_{X}^{2}}\right),
$$


where $d_{X}$ denotes the dimension of $X$. It suffices to check that

$$
\frac{1}{\operatorname{dim} \mathscr{D}_{X}} \sum_{Y \in D_{X}}\left|\frac{S_{X, Y}}{d_{X}}\right|^{2} \geq \frac{1}{d_{X}^{2}},
$$

since then (43) implies that $1 \leq\left(1+\operatorname{dim} \mathscr{T}_{X}\right) / d_{X}^{2}$, whence

$$
\operatorname{dim} \mathscr{T}_{X} \geq d_{X}^{2}-1
$$

But $X$ is noninvertible so $d_{X}>1$ and $\mathscr{T}_{X} \neq 0$.

Rewriting the left hand side of (44) as the sum of $\operatorname{dim} \mathscr{D}_{X}$ terms and using the inequality of arithmetic and geometric means we obtain

$$
\begin{aligned}
\frac{1}{\operatorname{dim} \mathscr{D}_{X}} \sum_{Y \in D_{X}}\left|\frac{S_{X, Y}}{d_{X}}\right|^{2} & =\frac{1}{\operatorname{dim} \mathscr{D}_{X}} \sum_{Y \in D_{X}} d_{Y}^{2}\left|\frac{S_{X, Y}}{d_{X} d_{Y}}\right|^{2} \\
& \geq \frac{1}{d_{X}^{2}}\left(\prod_{Y \in D_{X}}\left|\frac{S_{X, Y}}{d_{Y}}\right|^{2 d_{Y}^{2}}\right)^{1 / \operatorname{dim} \mathscr{D}_{X}} .
\end{aligned}
$$

The set $D_{X}$ is clearly stable under all automorphisms in the Galois group, and hence so is the product $\prod_{Y \in D_{X}}\left|S_{X, Y} / d_{Y}\right|^{2 d_{Y}^{2}}$. Therefore, this product belongs to $\mathbb{Q}$. Its factors are squares of absolute values of characters of $K_{0}(\mathscr{C})$ on $X$ and hence are algebraic integers. Since all factors are positive, the product is $\geq 1$, which implies (44).

For $X \in \mathbb{O}(\mathscr{C})$ define

$$
U_{X}=\left\{Y \in \mathscr{O}(\mathscr{C})|| S_{X, Y} \mid=d_{Y}\right\}
$$

Let $\varkappa_{X}$ be the full abelian subcategory of $\mathscr{b}$ generated by $U_{X}$.

Proposition 6.2. Let $\mathscr{C}$ be a weakly integral modular category and let $X$ be a simple noninvertible object in $\mathscr{C}$. Then

$$
3 \operatorname{dim} \mathscr{T}_{X}+\operatorname{dim} \boldsymbol{u}_{X}>\operatorname{dim} \mathscr{\ell} .
$$

Proof. We may assume $d_{X} \geq \sqrt{2}$.

We will use the following theorem of Siegel [1945] from number theory. Let $K / \mathbb{Q}$ be a finite Galois extension with the Galois group $G=\operatorname{Gal}(K / \mathbb{Q})$. Let $\alpha$ be a totally positive algebraic integer in $K, \alpha \neq 1$. Then

$$
\frac{1}{|G|} \sum_{\sigma \in G} \sigma(\alpha) \geq \frac{3}{2} \text {. }
$$


We apply this to the situation when $K$ is the extension of $\mathbb{Q}$ generated by entries of $S$. We compute

$$
\begin{aligned}
\operatorname{dim} \mathscr{C} & =\sum_{Y \in \mathscr{C}}\left|S_{X, Y}\right|^{2}=d_{X}^{2}+\sum_{Y \in U_{X}} d_{Y}^{2}+\sum_{Y \in \mathbb{O}(\mathscr{C})-\left(T_{X} \cup U_{X} \cup\{\mathbf{1}\}\right)}\left|S_{X, Y}\right|^{2} \\
& =d_{X}^{2}+\operatorname{dim} u_{X}+\sum_{Y \in \mathscr{O}(\mathscr{C})-\left(T_{X} \cup U_{X} \cup\{\mathbf{1}\}\right)} d_{Y}^{2}\left(\frac{1}{|G|} \sum_{\sigma \in G} \sigma\left(\frac{\left|S_{X, Y}\right|^{2}}{d_{Y}^{2}}\right)\right) \\
& \geq 2+\operatorname{dim} u_{X}+\frac{3}{2}\left(\operatorname{dim} \mathscr{C}-\operatorname{dim} \mathscr{T}_{X}-\operatorname{dim} u_{X}-1\right) ;
\end{aligned}
$$

therefore $3 \operatorname{dim} \mathscr{T}_{X}+\operatorname{dim} \mathfrak{U}_{X} \geq \operatorname{dim} \mathscr{b}+1>\operatorname{dim} \mathscr{b}$, as required.

Remark 6.3. Our proofs of Theorem 6.1 and Proposition 6.2 imitate the corresponding proofs for group characters given in [Berkovich and Zhmud' 1999].

\section{Acknowledgments}

We are grateful to P. Etingof, M. Müger, and V. Ostrik for useful discussions. Part of this work was done while Gelaki was on sabbatical in the Departments of Mathematics at the University of New Hampshire and MIT; he is grateful for their warm hospitality. His research was partially supported by the Israel Science Foundation (grant No. 125/05). Nikshych's research of was supported by the NSA grant H98230-07-1-0081 and the NSF grant DMS-0800545.

\section{References}

[Arkhipov and Gaitsgory 2003] S. Arkhipov and D. Gaitsgory, "Another realization of the category of modules over the small quantum group", Adv. Math. 173:1 (2003), 114-143. MR 2004e:17010 Zbl 1025.17004

[Bakalov and Kirillov 2001] B. Bakalov and A. Kirillov, Jr., Lectures on tensor categories and modular functors, University Lecture Series 21, American Mathematical Society, Providence, RI, 2001. MR 2002d:18003 Zbl 0965.18002

[Berkovich and Zhmud' 1999] Y. G. Berkovich and E. M. Zhmud', Characters of finite groups, vol. 2, Translations of Mathematical Monographs 181, American Mathematical Society, Providence, RI, 1999. MR 99j:20007 Zbl 0934.20009

[de Boer and Goeree 1991] J. de Boer and J. Goeree, "Markov traces and $\mathrm{II}_{1}$ factors in conformal field theory”, Comm. Math. Phys. 139:2 (1991), 267-304. MR 93i:81211 Zbl 0760.57002

[Bruguières 2000] A. Bruguières, "Catégories prémodulaires, modularisations et invariants des variétés de dimension 3”, Math. Ann. 316:2 (2000), 215-236. MR 2001d:18009 Zbl 0943.18004

[Coste and Gannon 1994] A. Coste and T. Gannon, "Remarks on Galois symmetry in rational conformal field theories", Phys. Lett. B 323:3-4 (1994), 316-321. MR 95h:81031

[Deligne 1990] P. Deligne, "Catégories tannakiennes", pp. 111-195 in The Grothendieck Festschrift, vol. 2, edited by P. Cartier et al., Progr. Math. 87, Birkhäuser, Boston, MA, 1990. MR 92d:14002 Zbl 0727.14010 
[Drinfeld et al. 2007] V. Drinfeld, S. Gelaki, D. Nikshych, and V. Ostrik, "Group-theoretical properties of nilpotent modular categories”, preprint, 2007. arXiv 0704.0195

[Drinfeld et al. 2009] V. Drinfeld, S. Gelaki, D. Nikshych, and V. Ostrik, "On braided fusion categories I", preprint, 2009. arXiv 0906.0620

[Etingof et al. 2005] P. Etingof, D. Nikshych, and V. Ostrik, "On fusion categories", Ann. of Math.

(2) 162:2 (2005), 581-642. MR 2006m:16051 Zbl 1125.16025

[Etingof et al. 2008] P. Etingof, D. Nikshych, and V. Ostrik, "Weakly group-theoretical and solvable fusion categories", preprint, 2008. arXiv 0809.3031

[Etingof et al. 2009] P. Etingof, D. Nikshych, and V. Ostrik, "Fusion categories and homotopy theory", preprint, 2009. arXiv 0909.3140

[Gaitsgory 2005] D. Gaitsgory, "The notion of category over an algebraic stack", preprint, 2005. arXiv 0507192

[Gelaki and Nikshych 2008] S. Gelaki and D. Nikshych, "Nilpotent fusion categories", Adv. Math. 217:3 (2008), 1053-1071. MR 2009b:18015 Zbl 1168.18004

[Ireland and Rosen 1990] K. Ireland and M. Rosen, A classical introduction to modern number theory, 2nd ed., Graduate Texts in Mathematics 84, Springer, New York, 1990. MR 92e:11001 Zbl 0712.11001

[Izumi 2001] M. Izumi, "The structure of sectors associated with Longo-Rehren inclusions, II: Examples”, Rev. Math. Phys. 13:5 (2001), 603-674. MR 2002k:46161

[Kassel 1995] C. Kassel, Quantum groups, Graduate Texts in Mathematics 155, Springer, New York, 1995. MR 96e:17041 Zbl 0808.17003

[Kirillov 2002] A. Kirillov, Jr., "Modular categories and orbifold models", Comm. Math. Phys. 229:2 (2002), 309-335. MR 2003m:17024 Zbl 1073.17011

[Majid 1991] S. Majid, "Representations, duals and quantum doubles of monoidal categories", Rend. Circ. Mat. Palermo (2) Suppl. 26 (1991), 197-206. MR 93c:18008 Zbl 0762.18005

[Montgomery 1993] S. Montgomery, Hopf algebras and their actions on rings, CBMS Regional Conference Series in Mathematics 82, Published for the Conference Board of the Mathematical Sciences, Washington, DC, 1993. MR 94i:16019 Zbl 0793.16029

[Müger 2000] M. Müger, "Galois theory for braided tensor categories and the modular closure", Adv. Math. 150:2 (2000), 151-201. MR 2001a:18008

[Müger 2003a] M. Müger, "From subfactors to categories and topology, I: Frobenius algebras in and Morita equivalence of tensor categories", J. Pure Appl. Algebra 180:1-2 (2003), 81-157. MR 2004f: 18013

[Müger 2003b] M. Müger, "On the structure of modular categories", Proc. London Math. Soc. (3) 87:2 (2003), 291-308. MR 2004g:18009

[Müger 2004] M. Müger, "Galois extensions of braided tensor categories and braided crossed $G$ categories", J. Algebra 277:1 (2004), 256-281. MR 2005b:18011

[Naidu and Nikshych 2008] D. Naidu and D. Nikshych, "Lagrangian subcategories and braided tensor equivalences of twisted quantum doubles of finite groups", Comm. Math. Phys. 279:3 (2008), 845-872. MR 2009b:16092 Zbl 1139.16028

[Nikshych 2008] D. Nikshych, "Non-group-theoretical semisimple Hopf algebras from group actions on fusion categories", Selecta Math. (N.S.) 14:1 (2008), 145-161. MR 2009k:16075 Zbl 05574000

[Ostrik 2003] V. Ostrik, "Module categories, weak Hopf algebras and modular invariants", Transform. Groups 8:2 (2003), 177-206. MR 2004h:18006 Zbl 1044.18004 
[Siegel 1945] C. L. Siegel, "The trace of totally positive and real algebraic integers", Ann. of Math. (2) 46 (1945), 302-312. MR 6,257a Zbl 0063.07009

[Tambara 2000] D. Tambara, "Representations of tensor categories with fusion rules of self-duality for abelian groups", Israel J. Math. 118 (2000), 29-60. MR 2001j:18015 Zbl 0969.18007

[Tambara 2001] D. Tambara, "Invariants and semi-direct products for finite group actions on tensor categories", J. Math. Soc. Japan 53:2 (2001), 429-456. MR 2002e:18010 Zbl 0980.18003

[Tambara and Yamagami 1998] D. Tambara and S. Yamagami, "Tensor categories with fusion rules of self-duality for finite abelian groups”, J. Algebra 209:2 (1998), 692-707. MR 2000b:18013 Zbl 0923.46052

[Turaev 2000] V. Turaev, "Homotopy field theory in dimension 3 and crossed group-categories", preprint, 2000. arXiv 0005291

[Turaev 2008] V. Turaev, "Crossed group-categories", Arab. J. Sci. Eng. Sect. C Theme Issues 33:2 (2008), 483-503. MR 2500054

Communicated by Susan Montgomery

Received 2009-05-21 Revised 2009-08-31 Accepted 2009-11-09

gelaki@math.technion.ac.il Department of Mathematics,

Technion-Israel Institute of Technology, 32000 Haifa, Israel

dnaidu@math.tamu.edu Department of Mathematics, Texas A\&M University, College Station, TX 77843, United States

nikshych@math.unh.edu

Department of Mathematics and Statistics, University of New Hampshire, Durham, NH 03824, United States 


\section{Algebra \& Number Theory}

www.jant.org

\section{EDITORS}

\section{MANAGING EDITOR}

Bjorn Poonen

Massachusetts Institute of Technology

Cambridge, USA

\author{
EDITORIAL BOARD CHAIR \\ David Eisenbud \\ University of California \\ Berkeley, USA
}

\section{BOARD OF EDITORS}

\section{Georgia Benkart}

Dave Benson

Richard E. Borcherds

John H. Coates

J-L. Colliot-Thélène

Brian D. Conrad

Hélène Esnault

Hubert Flenner

Edward Frenkel

Andrew Granville

Joseph Gubeladze

Ehud Hrushovski

Craig Huneke

Mikhail Kapranov

Yujiro Kawamata

János Kollár

Hendrik W. Lenstra

Yuri Manin

Barry Mazur
University of Wisconsin, Madison, USA

University of Aberdeen, Scotland

University of California, Berkeley, USA

University of Cambridge, UK

CNRS, Université Paris-Sud, France

University of Michigan, USA

Universität Duisburg-Essen, Germany

Ruhr-Universität, Germany

University of California, Berkeley, USA

Université de Montréal, Canada

San Francisco State University, USA

Hebrew University, Israel

University of Kansas, USA

Yale University, USA

University of Tokyo, Japan

Princeton University, USA

Universiteit Leiden, The Netherlands

Northwestern University, USA

Harvard University, USA
Susan Montgomery

Shigefumi Mori

Andrei Okounkov

Raman Parimala

Victor Reiner

Karl Rubin

Peter Sarnak

Michael Singer

Ronald Solomon

Vasudevan Srinivas

J. Toby Stafford

Bernd Sturmfels

Richard Taylor

Ravi Vakil

Michel van den Bergh

Marie-France Vignéras

Kei-Ichi Watanabe

Andrei Zelevinsky

Efim Zelmanov
University of Southern California, USA

RIMS, Kyoto University, Japan

Princeton University, USA

Emory University, USA

University of Minnesota, USA

University of California, Irvine, USA

Princeton University, USA

North Carolina State University, USA

Ohio State University, USA

Tata Inst. of Fund. Research, India

University of Michigan, USA

University of California, Berkeley, USA

Harvard University, USA

Stanford University, USA

Hasselt University, Belgium

Université Paris VII, France

Nihon University, Japan

Northeastern University, USA

University of California, San Diego, USA

\section{PRODUCTION}

ant@mathscipub.org

Paulo Ney de Souza, Production Manager

Silvio Levy, Senior Production Editor

See inside back cover or www.jant.org for submission instructions.

The subscription price for 2009 is US \$140/year for the electronic version, and \$200/year (+\$35 shipping outside the US) for print and electronic. Subscriptions, requests for back issues from the last three years and changes of subscribers address should be sent to Mathematical Sciences Publishers, Department of Mathematics, University of California, Berkeley, CA 94720-3840, USA.

Algebra \& Number Theory (ISSN 1937-0652) at Mathematical Sciences Publishers, Department of Mathematics, University of California, Berkeley, CA 94720-3840 is published continuously online. Periodical rate postage paid at Berkeley, CA 94704, and additional mailing offices.

ANT peer-review and production is managed by EditFLOw ${ }^{\mathrm{TM}}$ from Mathematical Sciences Publishers.

PUBLISHED BY

mathematical sciences publishers

http://www.mathscipub.org

A NON-PROFIT CORPORATION

Typeset in LATEX

Copyright $\odot 2009$ by Mathematical Sciences Publishers 


\section{Algebra \& Number Theory}

Volume $3 \quad$ No. $8 \quad 2009$

On coproducts in varieties, quasivarieties and prevarieties

GEORGE M. BERGMAN

Exponential sums nondegenerate relative to a lattice

Alan AdolphSON and STEVEN SPERBER

F-adjunction

KARL SCHWEDE

Log minimal models according to Shokurov

CAUCHER BIRKAR

Shlomo GELAKI, DEEPAK NAIDU and DMITRI NIKSHYCH 